\title{
Waste-Aware Single-Target Dilution of a Biochemical Fluid using Digital Microfluidic Biochips
}

\author{
Sudip Roy ${ }^{\mathrm{a}, *}$, Partha P. Chakrabarti ${ }^{\mathrm{b}}$, Krishnendu Chakrabarty $^{\mathrm{c}}$, Bhargab B. Bhattacharya $^{\mathrm{d}}$ \\ ${ }^{a}$ Department of Computer Science and Engineering, Indian Institute of Technology Roorkee, India, e-mail: sudiproy.fcs@iitrac.in \\ ${ }^{b}$ Department of Computer Science and Engineering, Indian Institute of Technology Kharagpur, India, e-mail: ppchak@cse.iitkgp.ernet.in \\ ${ }^{c}$ Department of Electrical and Computer Engineering, Duke University, Durham, USA, e-mail: krish@ee.duke.edu \\ ${ }^{d}$ Advanced Computing and Microelectronics Unit, Indian Statistical Institute, Kolkata, India, e-mail: bhargab@isical.ac.in
}

\begin{abstract}
A key challenge in design automation of digital microfluidic biochips is to carry out on-chip dilution/mixing of biochemical samples/reagents for achieving a desired concentration factor $(C F)$. In a bioassay, reducing the waste is crucial because the management of waste droplets is cumbersome and expensive; transporting them to the waste reservoir and washing their pathways to clean contaminated regions and remove the residue left by them may increase assay completion time. The existing dilution algorithms attempt to reduce the number of mix-split steps required in the process but focus little on minimization of sample requirement or waste droplets. In this work, we characterize the underlying combinatorial properties of waste generation and identify the inherent limitations of two earlier mixing algorithms twoWayMix [Thies et al., Natural Computing, 2008] and DMRW [Roy et al., TCAD, 2010] in addressing this issue. Based on these properties, we design an improved dilution/mixing algorithm (IDMA) that optimizes the usage of intermediate droplets generated during the dilution process, which in turn, reduces the demand of sample/reagent and production of waste. The algorithm terminates in $O(d)$ steps for producing a target $C F$ with a maximum error of $\frac{1}{2^{d+1}}$, where $d$ is the an accuracy level of the desired $C F$. Based on simulation results for all $C F$ values ranging from $\frac{1}{1024}$ to $\frac{1023}{1024}$ using a sample ( $100 \%$ concentration) and a buffer solution ( $0 \%$ concentration), we present an integrated scheme of choosing the best waste-aware dilution algorithm among four approaches such as twoWayMix, DMRW, REMIA [Huang et al., ICCAD, 2012] and IDMA, for a single target $C F$. Finally, an architectural layout of a DMF biochip that supports the proposed scheme is designed.
\end{abstract}

Keywords: Biochips, computer-aided-design, digital microfluidics, dilution, mixing, waste minimization.

\section{Introduction}

The recent emergence of digital microfluidic (DMF) biochips has led to a paradigm shift in many healthcare-related application areas, e.g., point-of-care clinical diagnostics, high5 throughput sequencing, toxicity monitoring and proteomics [26]. In DMF biochips, electrical actuation is normally used to manipulate (e.g., transport, merge, split, mix, dispense) discrete droplets of nanoliter or picoliter volume of sample/reagent flu-

\footnotetext{
A preliminary version of this paper appeared in the Proc. of IEEE/ACM DATE, 2011 [1].

${ }^{*}$ Corresponding author
}

ids on a two-dimensional array of electrodes [5, 7]. A schematic 10 biochip is shown in Fig. 11 [2]. Detailed descriptions and architectures of such biochips are available in the literature [2].

An efficient design of a microfluidic biochip mandates a complete integration of multiple assay operations such as detection, sample pre-treatment, sample mixing, and dilution control on one single chip [8]. The dilution problem arises in numerous biochemical assays for the preparation of samples or mixtures, e.g., cDNA for real-time PCR, immunoassays for detecting cy- 


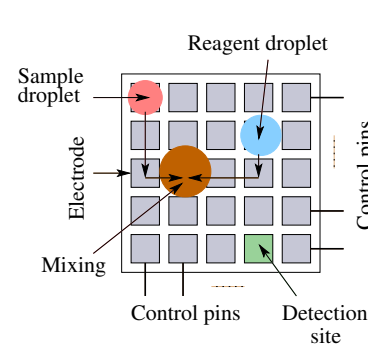

(a)

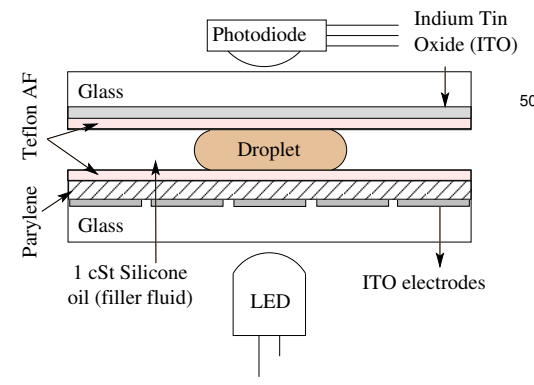

(b)
Figure 1: (a) Top view of a digital microfluidic biochip, and (b) Cross-sectional view of a cell at a detection site.

Note that larger the amount of waste-droplet production, higher will be the overhead for transporting them to the waste reservoir, and for washing their pathways to clean contaminated

regions and remove the residue therein. This may significantly impact assay completion time. The time needed for wastedroplet transportation, may however, be reduced by increasing the number of on-chip waste reservoirs, but again, this is not a good option. Hence, the reduction of waste droplets is desirable for lessening various on-chip droplet-transportation overheads of sample preparation algorithm will lead to minimum waste production.

Main Results: In this paper, we identify and analyze the inherent limitations of three existing dilution algorithms namely, twoWayMix [11], DMRW [12], REMIA [13], from the perspective of waste generation during the automation of single-target dilution procedure. We characterize the underlying combinatorial properties of discrete dilution process and waste generation. Based on these properties, we design a new dilution algorithm called Improved Dilution/Mixing Algorithm (IDMA) that maximizes the reuse of intermediate droplets generated during the process.

The proposed algorithm terminates in $O(d)$ steps for producing a target concentration factor $(C F)$ with a maximum error in $C F$ of $\frac{1}{2^{d+1}}$, where $d$ is the accuracy level of $C F$ and is user-defined. Given a target $C F$, we also propose an Integrated Dilution $\underline{\text { Scheme }}(I D S)$ for selecting the most efficient scheme from the perspective of waste reduction among twoWayMix, $D M R W, R E M I A$ and IDMA. In order to implement the integrated dilution scheme $I D S$, we design an architectural layout of electrodes for DMF-based biochips consisting of a $O(d)$-size rotary mixer, one merge-and-split type $(1: 1)$ mixer module, and a constant number of storage electrodes. The proposed methodology will help users in selecting the best solution for the automation of on-chip sample pre-processing.

The organization of the remainder of the paper is as follows. The related prior work on dilution algorithms and the motivating examples are discussed in Section 2 Section 3 discusses the proposed dilution algorithm along with related theoretical results. An integrated dilution scheme for single-target sample-preparation of two reactant fluids is presented in Sec- 
tion 4. An architectural layout of electrodes for implementing the proposed algorithm is described in Section 5. Simulation ${ }_{125}$ results of the integrated dilution scheme are presented in Section 6 Finally, the paper is concluded in Section 7 .

\section{Automated Dilution of Fluids: Prior Work and Motiva-} tion

\subsection{Prior Work}

DMF biochips typically deal with discrete droplets on a uniform 2D-array of equi-sized electrodes, hence their volumes are usually an integral multiple of that of a single droplet (unit volume). There are various $(k: \ell)$ mixing models that are often ${ }^{135}$ used, where $k$-unit volume of one substance is mixed with $\ell$-unit volume of another substance to produce $(k+\ell)$-unit volume of the resultant mixture in a single mixing operation. Three such cases are: (i) $k=\ell=1$, (ii) $k=\ell \neq 1$, and (iii) $k \neq \ell$, where $k$, $\ell$ are positive integers. The first case, i.e., $(1: 1)$ mixing model ${ }^{140}$ is easy to implement and hence it is widely used in practice.

The concentration factor $(C F)$ is defined as the ratio of the initial volume of the sample to the final volume of the diluted sample. Dilution is a special case of mixing two substances, where one of them is a buffer (neutral) solution with $C F=0$. If ${ }^{145}$ the samples of $C F C_{1}$ and $C_{2}$ are mixed in a volumetric ratio of $k: \ell$, then the resulting sample will have $C F C_{r}=\frac{k \cdot C_{1}+\ell \cdot C_{2}}{k+\ell}$, and a volume of $(k+\ell)$ units, assuming that the same buffer is used in diluting both the samples.

As mentioned earlier, in a DMF biochip, biochemical sam- ${ }^{150}$ ples can only be mixed using discrete volumes of liquid droplets. Therefore, it is possible to carry out dilution in only discrete steps to approximate the desired concentration level. In (1 : 1)-mixing model, two unit-volume droplets are mixed to obtain a new $C F$, and the resulting two-unit-volume droplet is ${ }^{155}$ split into two unit-volume droplets, out of which one is used in the next step of dilution and the other may not be used further (this is called a waste droplet). Thus, it is a challenge to achieve a desired $C F$ using the fewest mix-split steps. The dilution algorithm reported by Griffith et al. [14] outputs a se- ${ }^{160}$ quence of $(1: 1)$ mix-split steps to be performed in order to achieve a certain target $C F$. Thies et al. [11] reported a dilution/mixing algorithm for minimizing the total number of mixsplit steps. Subsequently, many other algorithms with various objectives appeared in the literature for automated sample preparation [11, 15-18]. In general, the goal in these algorithms is to minimize the number of mix-split steps (i.e., mixing time), reactant usage, storage requirement, or waste production. In some approaches, the same cost (per unit volume) is assigned to both sample and buffer fluids [1, 12, 19, 20]. These algorithms are primarily focused on the minimization of waste droplets, which indeed minimizes the total cost of input fluids (sample and buffer). In another important approach to sample preparation, different costs are assigned to various input reactant fluids. Based on this variable-cost metric, several algorithms for minimization of reactant usage were reported [13, 21-24].

The state-of-the-art dilution algorithms can be broadly classified into two categories: (a) single-target dilution algorithms and (b) multi-target dilution algorithms (in which droplets of different concentrations need to be prepared at the same time). Most of the earlier work on single-target dilution aim to minimize the number of mix-split steps, wastage or reactant usage. These challenges become more critical for the multi-target dilution problem because an optimal sharing of intermediate droplets (some of which would have been otherwise wasted), is needed for the reduction of time and cost of the overall procedure. During the last few years, several heuristic approaches were proposed in the literature for both single and multi-target sample preparation problems [13, 15, 19,-22, 24,-26].

Hsieh et al. proposed two algorithms namely, IDSA [21] and RSM [22], for preparing multiple targets to achieve waste minimization. Mitra et al. [15] reported a dilution algorithm based on de Bruijn graphs for generating droplets with multiple $C F$ s without using any intermediate storage. Huang et al. [13] introduced an algorithm called REMIA with an objective of minimizing reactant usage as well as wastage for solving the multi-target dilution problem. Bhattacharjee et al. [19, 25] proposed a pruning-based dilution algorithm to reduce wastage during the generation of diluted sample with multiple concen- 
trations.

Another multi-target sample preparation algorithm is proposed by Huang et al. [26], which is referred to as WARA. It extensively exploits the ideas of waste recycling and intermediate droplet sharing to reduce both reactant usage and wastage. Note that the algorithm WARA reduces to REMIA [13] for the single-target dilution problem. Liu et al. [24] presented an al-195 gorithm namely Ext-CoDOS for multi-target dilution problem, which identifies the possibilities of sharing two common dilution operations for reactant minimization. Dinh et al. [20] considered the objective of reducing reactant usage and waste production in one model. They proposed an algorithm based ${ }^{200}$ on a network-flow model, which can be tuned to optimize the desired objective. It is capable of optimizing the cost of reactants and loss of waste droplets even in a multi-target sample preparation scenario.

Another important practical issue was stated as an open ${ }^{205}$ problem by Thies et al. [11]: if two inputs are related (e.g., a sample of $10 \%$ acid and a sample of $20 \%$ of the same acid), then how to produce a desired dilution of the sample (say, 17\%). Most of the existing algorithms consider the problem of automated dilution of a fluid from the supply of two pure $(100 \%)^{210}$ input fluids and they do not discuss generalized dilution from multiple arbitrary concentrations. Some of the recently published work considered this issue and provided a solution [1, 12, 14, 29]. Table 1 indicates the scope of the proposed work against earlier approaches, where the entry "Yes/No" indicates ${ }^{215}$ the applicability of the concerned method ( $T_{m s}$ is the total num-

Table 1: Scope and results of the proposed work compared to prior art.

\begin{tabular}{|c|c|c|c|c|c|}
\hline \multirow{2}{*}{$\begin{array}{c}\text { Dilution } \\
\text { Algorithm }{ }^{\dagger}\end{array}$} & \multirow{2}{*}{$\begin{array}{c}\text { \# Target } C F \mathrm{~s} \\
\text { (\# Droplets) }\end{array}$} & \multicolumn{3}{|c|}{ Minimization Criteria } & \multirow{2}{*}{$\begin{array}{l}\text { Arbitrary } \\
\text { Input } C F\end{array}$} \\
\hline & & $T_{m s}$ & Wastage & Reactant Usage & \\
\hline Griffith et al. 14] & Single (2) & No & No & No & Yes \\
\hline twoWayMix [11] & Single (2) & Yes & No & No & No \\
\hline$D M R W[12$ & Single (2) & No & Yes & No & Yes \\
\hline Roy et al.[1] & Single (2) & No & Yes & No & Yes \\
\hline \begin{tabular}{ll|l|l|l|l|l|l|l|l|l|l|} 
& 18 & 27 \\
\end{tabular} & Single $(\geq 2)$ & Yes & No & No & No \\
\hline [13. 21,24 & One or More (2) & No & No & Yes & No \\
\hline \begin{tabular}{|l|l|}
$D B G A$ & 28 \\
\end{tabular} & Multiple (2) & Yes & No & No & No \\
\hline PBDA 19 & Multiple (2) & No & Yes & No & No \\
\hline WARA 26 & Multiple (2) & No & Yes & Yes & No \\
\hline$N F S P$ 20 & One or More $(\geq 2)$ & No & Yes & Yes & No \\
\hline \begin{tabular}{|l|l|l|l|l|l|}
$G D A, E G D A$ & 30 \\
\end{tabular} & Single (2) & Yes & No & No & Yes \\
\hline Proposed Scheme & Single (2) & Yes & Yes & No & Yes \\
\hline
\end{tabular}

ber of (1:1) mix-split steps required to produce target droplets).

\subsection{Motivation of the Work}

In this paper, we focus only on the single-target dilution problem and assume that both sample and buffer solution have the same cost per unit volume. Hence, in this case, waste minimization will automatically minimize the total cost of reactants used. We consider twoWayMix [11], DMRW [12], and REMIA [13] as three representative algorithms for waste minimization, and use them in our comparative study.

Note that after one $(1: 1)$ mix operation, the $C F$ of the mixture becomes the mean value of those of the two source droplets. Thus, for the sake of convenience, the $C F$ of a target dilution $\left(C_{t}, 0 \leq C_{t}<1\right)$, is expressed as a $d$-bit binary fraction, when an accuracy level of $d$ is desired. In other words, the $C F$ of a target droplet can be expressed as $\frac{x}{2^{d}}$, where $x \in$ $\mathbb{Z}^{+}, 0 \leq x \leq\left(2^{d}-1\right)$. Also, a buffer solution can be assumed to have $C F=0\left(=\frac{0}{2^{d}}\right)$, and a pure sample can be assumed to have $C F=1\left(=\frac{2^{d}}{2^{d}}\right)$. For our study, we assume that $d=10$, and a sample ( $100 \%$ conc.) and a buffer ( $0 \%$ conc.) are given; hence, a target $C F$ value that lies between $\frac{1}{1024}$ to $\frac{1023}{1024}$ will be of interest to us.

We first analyze the performance of two dilution algorithms namely, twoWayMix [11] and DMRW [12], for several CF s over the range from $\frac{1}{1024}$ to $\frac{1023}{1024}$. The twoWayMix always uses either sample or buffer solution at each $(1: 1)$ mix-split step to mix with the current droplet. Thus, it consumes only one droplet at every mix-split step to continue the process, and produces one waste droplet at each step (excepting the last one, where the target dilution is achieved). For example, the mix-split procedure for the target $C F C_{t}=\frac{341}{1024}$ for the twoWayMix is illustrated in Fig. 2. where the number of waste droplets is nine.

Note that this sequence of mix-split steps can be conveniently represented by a directed graph as shown in Fig. 2[12]. Each node of this graph represents a $C F$ value; the top and bottom nodes represent the sample $\left(C F=1=\frac{1024}{1024}\right)$ and the buffer $\left(C F=0=\frac{0}{1024}\right)$ respectively. These two nodes have in-degree zero. The node with out-degree zero (in this case $\frac{341}{1024}$ ) rep- 
resents the target $C F$. Other nodes have in-degree two, and out-degree one, each. If a node $v_{i}$ has two predecessor nodes $v_{j}$ and $v_{k}$, one of them (say, $v_{j}$ ) must be either the sample node or the buffer node according to the requirement of two WayMix 260 algorithm; the other predecessor node $\left(v_{k}\right)$ indicates a droplet of $C F=v_{k}$ produced at the last dilution step, and a $(1: 1)$ mixture of $v_{j}$ and $v_{k}$ produces two droplets of $C F=v_{i}$. For example, the node $\left(\frac{640}{1024}\right)$ has two predecessor nodes $\left(\frac{256}{1024}\right)$ and $\left(\frac{1024}{1024}\right)$; the two directed edge towards the node $\left(\frac{640}{1024}\right)$ represents the ${ }_{265}$ fact that one droplet of $C F=\frac{256}{1024}$ is mixed with one droplet of $C F=\frac{1024}{1024}$, and then the mixture is split to produce two droplets of intermediate $C F=\frac{640}{1024}$. As only one of the resulting droplets is needed in the next step, one droplet is wasted at each step but the last one (denoted by a black dot adjacent to a node).

In the twoWayMix algorithm, each droplet of an intermediate $C F$ value is required only once in the mix-split sequence, whereas in the $D M R W$ algorithm, it may be needed multiple times in order to reach a target $C F$. Thus, to meet the demand of intermediate $C F$ values, these droplets are to be produced 275 starting from the given supply of sample and buffer fluids. As a result, $D M R W$ may need a larger number of $(1: 1)$ mix-split steps compared to twoWayMix; however, the number of waste droplets is reduced significantly. In order to implement $D M R W$, a layout architecture with a rotary mixer was used for perform-280 ing $(k: k)$ mixing $\left(k \geq 1\right.$, where $\left.k \in \mathbb{Z}^{+}\right)$in one step [12].

In the $D M R W$ method, it is assumed that a sample with two initial $C F \mathrm{~s}, C_{\ell}$ and $C_{h}$, is supplied such that and the desired target $C F C_{t}$ satisfies $0 \% \leq C_{\ell} \leq C_{t}<C_{h} \leq 100 \%$. Initially, $C_{\ell}$ and $C_{h}$ are considered as the lower- and upper-boundary $C F \mathrm{~S}_{285}$ respectively. At every mix-split step, it always mixes only two

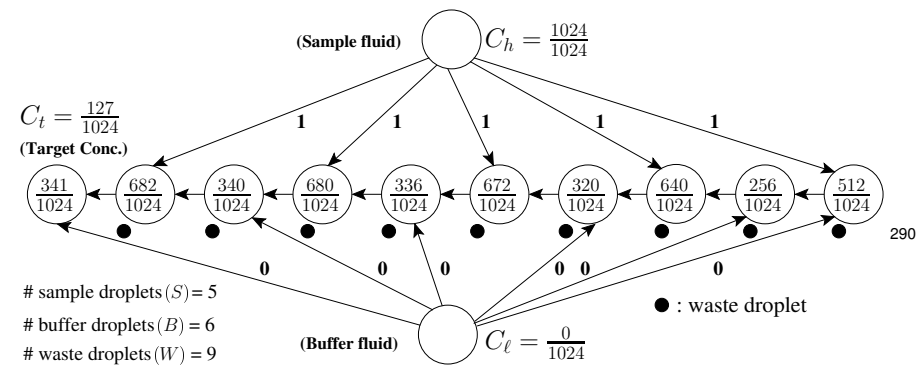

Figure 2: Mix-split steps obtained by twoWayMix for the target $C F C_{t}=\frac{341}{1024}(\equiv$ $\left.0.0101010101_{2}\right)$. boundary $C F$ s. After such a $(1: 1)$ mixing, it compares the resulting $C F\left(C_{i}\right)$ with $C_{t}$. If they are equal within the error margin of $\frac{1}{2^{d}+1}$, the target $C F$ is said to have reached; otherwise, if $C_{i}$ is less (greater) than $C_{t}$, then we replace the lower-boundary (upper-boundary) $C F$ by $C_{i}$. This process is repeated until the target $C F C_{t}$ is reached. Note that at every mix/split step, the interval (i.e., the range of $C F \mathrm{~s}$ ) between the lower and upper boundaries not only includes the target $C F$, but also continues to shrink; it finally converges to the target $C F$ satisfying the desired error margin within at most $d$ steps.

As before, the complete mix-split sequence $Z_{m}, m \leq d$, that leads to the target $C F$ can be conveniently depicted using a weighted digraph $\mathcal{M}(V, E)$, where a node $v \in V$ denotes a $C F$ value. The edge set $E$ is defined as follows: when a $1 X$-volume droplet of $C F=C_{i}$ is mixed with another $1 X$-volume of droplet of $C F=C_{j}$ to produce a mixture of $C F C_{k}$ (i.e., $C_{k}=\frac{C_{i}+C_{j}}{2}$ ), we represent this fact by putting two directed edges in the graph $\left(v_{i}, v_{k}\right)$ and $\left(v_{j}, v_{k}\right)$.

For example, consider the mix-split digraph for the target $C F=\frac{127}{1024}$ shown in Fig. 3 (a). Here, the initial boundary $C F$ s, which are supplied, are $\frac{0}{1024}$ and $\frac{1024}{1024}$. Note that for the sake of clarity of the diagram, the nodes in the digraph are not shown as explicit bubbles; they are implicitly assumed to be present at the two ends of every edge. In this digraph, the initial boundary nodes have in-degree zero and every other node has in-degree exactly two. Only the target node has out-degree zero; the outdegree of other nodes may lie between 1 to $d$. In $D M R W$, the digraph $\mathscr{M}$ representing the mix-split sequence $Z_{m}$ for a target $C F$ is usually drawn in a special manner to reflect gradual shrinkage of the bounding interval with respect to the target; for example, in Fig. 3 a), we mix $\frac{0}{1024}$ and $\frac{1024}{1024}$ to produce $\frac{512}{1024}$; this is shown by the two directed edges incident on it from them. Imagine a virtual vertical line that passes through the target node. Since the intermediate $C F\left(\frac{512}{1024}\right)$ is higher than the target $C F$, the node $\frac{512}{1024}$ is drawn on the right side of the virtual line. In the next step, $\frac{256}{1024}$ is produced by mixing the two current boundary $C F$ s $\left(\frac{0}{1024}\right.$ and $\left.\frac{512}{1024}\right)$, and this process is continued until the target is reached. A directed edge incident on a node $i$ from the 


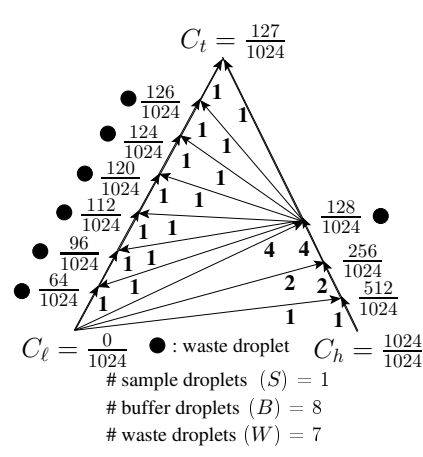

(a)

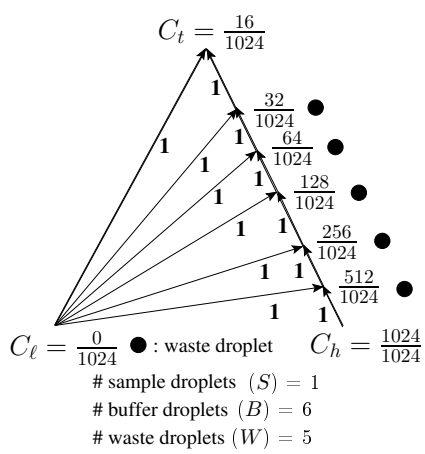

(c)

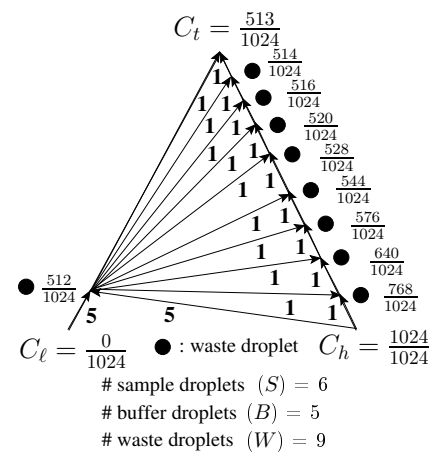

(b)

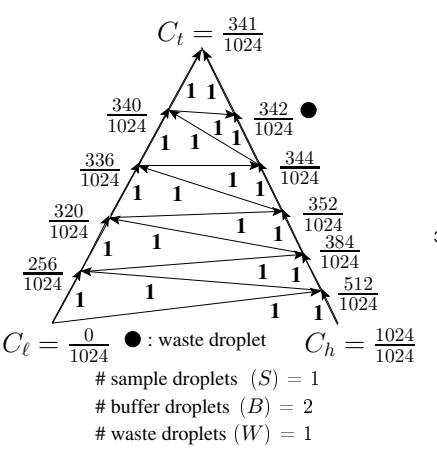

(d)
Figure 3: Digraphs obtained by DMRW [12] for the target $C F$ (a) $C_{t}=\frac{127}{1024}$, (b) $C_{t}=\frac{513}{1024}$, (c) $C_{t}=\frac{16}{1024}$, and (d) $C_{t}=\frac{341}{1024}$, using the supply of droplets with $C F$ s $C_{\ell}=\frac{0}{1024}$ and $C_{h}=\frac{1024}{1024}$.

(left) right predecessor is called a (left-) right-edge of $i$.

The sequence of intermediate $C F$ values that leads to the target from the two initial boundary values is given by the longest directed path (i.e., of length $m$ ) to the target in $\mathcal{M}$. Also, from a visual perspective, the $C F$-values correspond- via $C_{t}$, appear in an ascending order when the graph is embedded on a plane. For example, in Fig. 3(b) this order is $\frac{0}{1024}, \frac{256}{1024}, \frac{320}{1024}, \ldots, \frac{341}{1024}, \frac{342}{1024}, \frac{344}{1024}, \ldots, \frac{1024}{1024}$. For some other example target $C F$ s $C_{t}=\frac{513}{1024}, C_{t}=\frac{16}{1024}$ and $C_{t}=\frac{341}{1024}$, the corresponding mix-split sequences (or digraphs), the number of sample, buffer, and waste droplets are shown in Fig. 3. b) -3. d), respectively.

Note that with each directed edge $\left(v_{i}, v_{j}\right)$ of $\mathcal{M}$, an integer weight $D(i)$ is attached; this is indicative of how many $1 X-345$ volume droplets, with $C F$ value denoted by the node $v_{i}$, are to be produced to complete the dilution process. These weights can be found by retracing the graph from the target node $C_{t}$,

where two $1 X$ droplets will be produced at the end [12]. In this context, we define the skew of a node as follows. The skew run (Fig. 2). Also, it is easy to note that the weight of a directed edge $\left(v_{i}, v_{j}\right)$ is equal to $D(i)=\left\lceil\frac{S k(i)}{2}\right\rceil$, if we assume a $(k: k)$ mixing model.

A close look at Figs. 3 a)-3 (d) reveals that the skew of a node representing an intermediate $C F$ in the digraph has a strong impact on waste droplet generation as well as on the total number of $(1: 1)$ mix-split steps. This follows from the fact that in each $(1: 1)$ mix-split step, two droplets of an intermediate $C F$ are produced and therefore, the demand of this node as well as wastage is optimized, when both of these two droplets are consumed in the subsequent mix-split steps. The above observation motivates us to further reduce the number of waste droplets generated in $D M R W$ [12], by controlling the skew of an intermediate $C F$ s node.

\section{Proposed Dilution Algorithm: IDMA}

We now present a new heuristic-based dilution algorithm, called Improved Dilution/Mixing Algorithm (IDMA) for waste minimization based on the $D M R W$ approach. Assume that we are supplied with two input fluids, one with lower $C F C_{\ell}$ and another with higher $C F C_{h}$. The objective is to produce a target droplet of $C F C_{t}$ with an accuracy level of $d$, where $0 \leq C_{\ell} \leq C_{t}<C_{h} \leq 1$. First, DMRW is run to construct the digraph $\mathcal{M}$ corresponding to the sequence $Z_{m}$ of $m(k: k)$ mixsplit steps, $m \leq d$. The execution of IDMA needs the following 
1. Approximate $C_{t}$ as $\frac{c}{2^{d}}$ for the accuracy level $d$, where $c, d$ are positive integers with $0 \leq c<2^{d}$ and $d \geq 1 . \quad 375$

2. Represent $C_{\ell}$ as $\frac{a}{2^{d}}$ and $C_{h}$ as $\frac{b}{2^{d}}$, where $a, b$ are positive integers with $0 \leq a<b \leq 2^{d}$.

Table 2 lists the notation used in this paper. Note that the workload of one $(k: k)$ mix-split step can be assumed to be that of $k(1: 1)$ mix-split steps. Thus, $T_{m s}$ for a $(k: k)$ mix-split step is computed as accordingly.

For any digraph, we do the following steps to compute the performance parameters.

1. Set the skew of $\operatorname{node}\left(C_{t}\right)$ as two, i.e., $\operatorname{Sk}\left(\operatorname{node}\left(C_{t}\right)\right)=2$.

2. Backtrace the digraph in reverse topological order starting from node node $\left(C_{t}\right)$. For each node $v_{i}$, consider the left-edge $\left(v_{\ell}, v_{i}\right)$ and the right-edge $\left(v_{r}, v_{i}\right)$ incident on $v_{i}$; set their weights $\operatorname{In}_{\ell}(i)=\operatorname{In}_{r}(i)=\left\lceil\frac{S k(i)}{2}\right\rceil$.

3. For each edge $\left(v_{i}, v_{j}\right)$, compute the edge-weight $D(i)$, i.e., the number of droplets of $C F(i)$ to be produced, as $D(i)=\operatorname{In}_{\ell}(i)+\operatorname{In}_{r}(i)=2\left\lceil\frac{S k(i)}{2}\right\rceil$, whereas the number of droplets of $C F(i)$ used subsequently is $S k(i)$. Thus, the number of waste droplets with $C F(i)$ can be obtained as $W(i)=D(i)-S k(i)$. Note that for any node $i, W(i)$ is only 1 or 0 .

Table 2: Notation used in this paper.

\begin{tabular}{|c|c|}
\hline Symbols & Meaning \\
\hline$Z_{m}$ & Sequence of $m$ mix-split steps $(m \leq d)$ obtained by $D M R W$ \\
\hline $\mathcal{M}$ & Digraph or dilution tree representing $Z_{m}$ \\
\hline$Z_{m^{\prime}}$ & $\begin{array}{l}\text { Modified sequence of } m^{\prime} \text { mix-split steps obtained by } \\
\text { IDMA }\end{array}$ \\
\hline $\mathcal{M}^{\prime}$ & Modified digraph representing $Z_{m}^{\prime}$ obtained by IDMA \\
\hline$N\left(C_{i}\right)$ & Node in a digraph corresponding $C F=C_{i}$ \\
\hline$C F(i)$ & $C F$ corresponding to a node $i$ in the digraph \\
\hline CFleft $(i)$ & $C F$ of the left predecessor of the node $i$ in a digraph \\
\hline CFright $(i)$ & $C F$ of the right predecessor of the node $i$ in a digraph \\
\hline$S k(i)$ & Skew of a node $i$ in a digraph \\
\hline$S k_{\max }$ & Maximum skew of any node in a digraph \\
\hline$D(i)$ & $\begin{array}{l}\text { The integer weight associated with a directed edge }\left(v_{i}, v_{j}\right) \text {, } \\
\text { i.e., the number of droplets to be produced whose } C F \text { is } \\
\text { denoted by the node } v_{i} \text { in a mix-split digraph }\end{array}$ \\
\hline $\operatorname{In}_{r}(i)\left(I_{\ell}(i)\right)$ & Weight attached to the right(left)-edge incident on node $i$ \\
\hline$O(i)$ & Out-degree of a node $i$ in a digraph \\
\hline$U(i)$ & $\begin{array}{l}\text { Number of demanded droplets of the } C F \text { denoted by node } \\
i \text { in a digraph }\end{array}$ \\
\hline$T_{m s}$ & Total number of $(1: 1)$ mix-split steps in a digraph \\
\hline$W$ & Total number of waste droplets generated \\
\hline$W(i)$ & $\begin{array}{l}\text { Number of waste droplets with the } C F \text { denoted by the } \\
\text { node } i \text { in a digraph }\end{array}$ \\
\hline$W L_{\ell}$ & Workload on the linear mixer capable of $(1: 1)$ mixing \\
\hline$W L_{r}$ & Workload on the rotary mixer capable of $(k: k)$ mixing \\
\hline
\end{tabular}

4. The total number of waste droplets generated is $W=\sum_{i} W(i)$.

5. Scan the digraph to compute the total number of $(1: 1)$ mix-split steps as $T_{m s}=\sum_{i} \operatorname{In}_{\ell}(i)$.

\subsection{Algorithm Overview: ImproveDigraph}

The proposed dilution algorithm IDMA uses a heuristicbased procedure ImproveDigraph, which first modifies the digraph $\mathcal{M}$ obtained by $D M R W$ [12]. Let $\mathcal{M}^{\prime}$ be the modified digraph corresponding to the modified sequence $Z_{m^{\prime}}$ of $m^{\prime}(k: k)$ mix-split steps obtained by ImproveDigraph, where $m^{\prime} \geq m$. Note that in $D M R W$, if the intermediate $C F$ values appear alternately on the left (right) and right (left) side with respect to the target $C F$ as in Fig. 3(d), the skew of these nodes will be exactly two. In these cases, both the droplets generated at a node are consumed without any wastage. Thus, when $S k(i)$ of a node $i$ is greater than two, we attempt to find a modified bounding interval by changing the lower-boundary $C F$ (or the upper-boundary $C F$ ) depending on the target $C F$ value. In order to achieve this, a few mix-split steps are executed starting from the current boundary values to produce the new boundary $C F$. We write a procedure SkewModify(skNode) that performs this task to modify a digraph, in which the node $s k N o d e$ is the first node in the mix-split sequence graph obtained by $D M R W$ whose skew $S k>3$. This simple heuristic applied on the digraph obtained from $D M R W$ can significantly reduce both $W$ and $T_{m s}$. The procedure ImproveDigraph is described as Algorithm 1 ,

Algorithm ImproveDigraph preserves the same accuracy level of $d$ in the target $C F$ as in DMRW. Since the execution of $D M R W$ requires $O(d)$ time, the overall time complexity of ImproveDigraph is also $O(d)$. For an illustration of ImproveDigraph, consider the digraphs of Fig. 3(a) and Fig. 3.b) obtained by the $D M R W$ method for two target $C F \mathrm{~s} C_{t}=\frac{127}{1024}$ and $C_{t}=\frac{513}{1024}$; when Algorithm $\mathbf{1}$ is run, we get two two modified digraphs as shown in Fig. 4(a) and 4(b), respectively. Note that in Fig. 4 a), we generate a modified upper-boundary $C F\left(\frac{192}{1024}\right)$, and in Fig. 4(b), we modify the lower-boundary $C F$ to $\left(\frac{384}{1024}\right)$. 

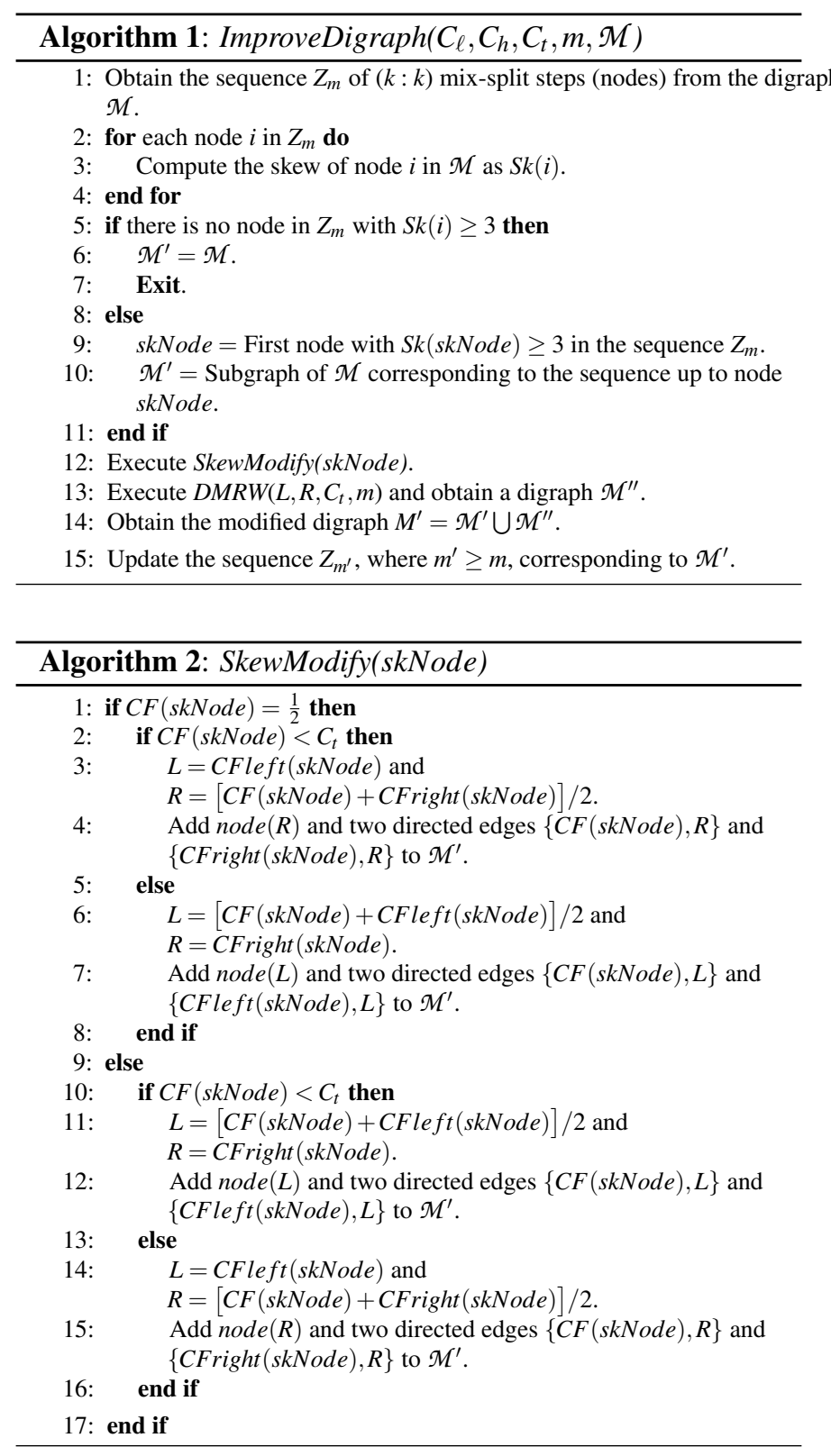

In each case, both the number of waste droplets $(W)$ and the number of (1:1) mix-split steps $\left(T_{m s}\right)$ are reduced compared to $D M R W$; compared to twoWayMix, W is also reduced. However, for the target $C F \mathrm{~s}$, such as $\frac{1}{1024}$ or $\frac{1023}{1024}$, the digraph obtained by $D M R W$ has high skew value on the two input $C F \mathrm{~s}(0 \%$ conc. and $100 \%$ conc.) and hence, the proposed scheme ImproveDigraph cannot improve $W$ or $T_{m s}$. Another extreme case is target $C F \frac{16}{1024}$, for which the digraph obtained by $D M R W$ (Fig. 3 (c)) cannot be improved by ImproveDigraph. Here, the node with $C F C_{\ell}=\frac{0}{1024}$ has the skew value $S k\left(C_{\ell}\right)=6$ and the two meth-

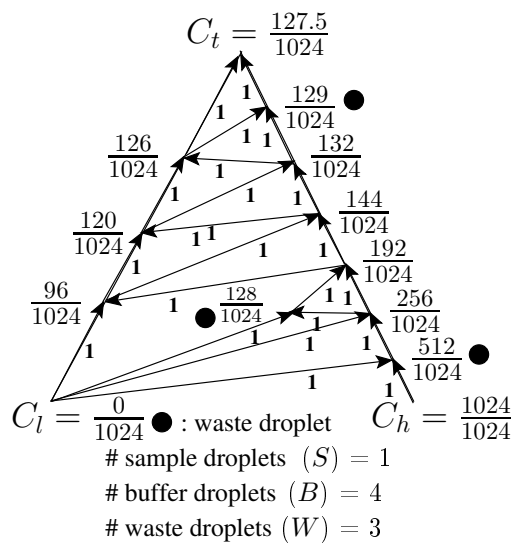

(a)

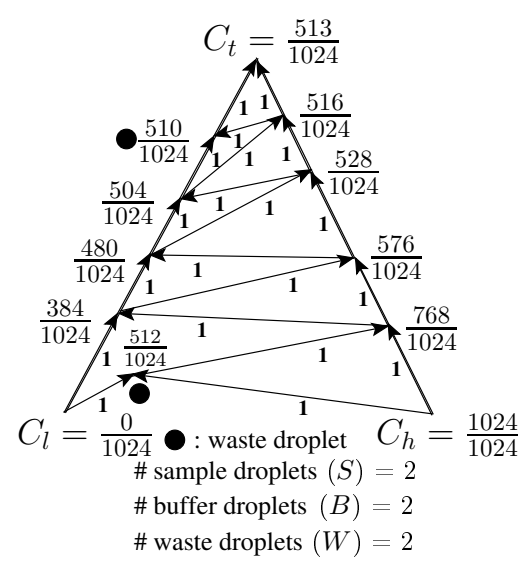

(b)

Figure 4: Digraphs obtained by ImproveDigraph for (a) $C_{t}=\frac{127}{1024}$ and (b) $C_{t}=$ $\frac{513}{1024}$.

of $W$ and $T_{m s}$.

\subsection{Theoretical Analysis}

Consider the target $C F \frac{341}{1024}$, for which ten (1:1) mixsplit steps are required by $D M R W$ as shown in Fig. 3.d). Note that out of ten mix-split steps executed in this case, a droplet is wasted only in one step. This observation motivates us to analyze the combinatorial properties of a mix-split sequence, which are responsible for the production of waste droplets. This analysis also leads to a powerful heuristic called "one-third rule", which is used in Algorithm 1 .

Note that in the mix-split digraph obtained by $D M R W$, if the progression of the sequence towards the target $C F$ alternates between the left (right) and right (left) side of the target, then the out-degree of each node can never exceed two, and therefore, ${ }_{435}$ the number of waste droplets is optimized and no additional demand of intermediate $C F \mathrm{~s}$ is created. Our analysis reveals 
that such an alternating mix-split sequence is possible, resulting in two target droplets of $C F=C_{t}$ with only one waste droplet. For example, if $d=10, C_{\ell}=\frac{0}{2^{d}}$ and $C_{h}=\frac{2^{d}}{2^{d}}$, this happens for

$C_{t}=\frac{341}{1024}$ or $C_{t}=\frac{683}{1024}$. These two target $C F \mathrm{~s}$ correspond to the case where a sample is mixed with a buffer in a ratio of $(1: 2)$, or (2: 1). In other words, the number 341 (683) appears nearly at the one-third (two-third) position of the interval between 0 to 1024 (see Fig. 5).

It may be observed that, if the target $C F$ is at one-third position of the interval $\left(C_{h}-C_{\ell}\right)$, i.e., $\frac{b-a}{2^{d}}$, where $C_{h}=\frac{b}{2^{d}}$ and $C_{\ell}=\frac{a}{2^{d}}$, then during the next mix-split step (in the sequence of alternate steps) the input fluid droplet with lower $C F\left(C_{\ell}\right)$ is mixed with the resultant droplet of the previous step to get a new intermediate $C F$ (Case-I). On the other hand, if the target ${ }^{465}$ $C F$ is at two-third position of the interval $\left(C_{h}-C_{\ell}\right)$, then during the next mix-split step the input fluid droplet with higher $C F\left(C_{h}\right)$ is mixed with the resultant droplet of the previous step to get the new intermediate $C F$ (Case-II). An alternating mixsplit steps are then executed until the target $C F$ is reached. The result can be generalized in the form of the following theorem.

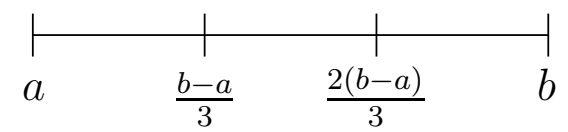

Figure 5: Scale of the input $C F$ s $C_{\ell}=\frac{a}{2^{d}}$ and $C_{h}=\frac{b}{2^{d}}$.

Theorem 1. Starting from two boundary CFs $C_{\ell}=\frac{a}{2^{d}}$ and $C_{h}=$ $\frac{b}{2^{d}}$, where $0 \leq C_{\ell}<C_{h} \leq 1$, an alternating sequence of $i(1: 1)$ mix-split steps will lead to two droplets of target $C F C_{i}=\frac{c_{i}}{2^{d}}$ with only one waste droplet, where:

$$
c_{i}=\frac{1}{3}\left[\left(2 a+\frac{(-1)^{i+1}}{2^{i+1}} a\right)+\left(b+\frac{(-1)^{i}}{2^{i+1}} b\right)\right](\mathbf{C a s e}-\mathbf{I})
$$

or

$$
c_{i}=\frac{1}{3}\left[\left(a+\frac{(-1)^{i}}{2^{i+1}} a\right)+\left(2 b+\frac{(-1)^{i+1}}{2^{i+1}} b\right)\right](\text { Case }- \text { II })
$$

where $i$ is a non-negative integer ranging from 0 to $\left(\left\lfloor\log _{2}(b-\right.\right.$ a) $\rfloor-1)$.

Proof. Theorem 1 can be proved by solving a recurrence re- resultant $C F$ s obtained by the $D M R W$ method can be written as

$$
\begin{gathered}
c_{i}=\frac{1}{2}\left(c_{i-1}+c_{i-2}\right) \\
\therefore 2 c_{i}-c_{i-1}-c_{i-2}=0
\end{gathered}
$$

We consider Case-I first, i.e., $C_{\ell}$ is mixed with $C_{0}$ to obtain $C_{1}$. Thus, the initial conditions are: $c_{0}=\frac{a+b}{2}$ and $c_{1}=\frac{1}{2}\left(\frac{a+b}{2}+\right.$ a) $=\frac{3 a+b}{4}$.

Next, we solve this recurrence to prove that Eqn. (1) is true. Let $c_{i}=r^{i}$ be a ("guess") solution of the recurrence relation in Eqn. (3) for some undetermined value of $r$. Then, we have the equation

$$
2 r^{2}-r-1=0
$$

as the characteristic equation for the homogeneous recurrence relation in Eqn. (3) and the two distinct roots of this equation are the characteristic roots given by $r_{1}=1$ and $r_{2}=-\frac{1}{2}$. So, the general solution to the recurrence relation can be written as

$$
\begin{aligned}
c_{i} & =\alpha_{1}\left(r_{1}\right)^{i}+\alpha_{2}\left(r_{2}\right)^{i} \\
\therefore c_{i} & =\alpha_{1}(1)^{i}+\alpha_{2}\left(-\frac{1}{2}\right)^{i}
\end{aligned}
$$

where $\alpha_{1}$ and $\alpha_{2}$ are real constants.

From Eqn. (4), we can determine the values of $c_{0}$ and $c_{1}$. To find $\alpha_{1}$ and $\alpha_{2}$ we plug-in the initial conditions to Eqn. (4):

$$
\begin{array}{r}
\alpha_{1}+\alpha_{2}=\frac{a+b}{2} \quad(\text { putting } i=0) \\
\text { and } \alpha_{1}+\alpha_{2} \cdot\left(-\frac{1}{2}\right)=\frac{3 a+b}{4} \quad(\text { putting } i=1)
\end{array}
$$

Solving these equations we get the values as $\alpha_{1}=\frac{2 a+b}{3}$ and $\alpha_{2}=\frac{-(a-b)}{6}$. Thus, we get an exact formula for $c_{i}$ as follows:

$$
\begin{aligned}
c_{i} & =\alpha_{1}+\alpha_{2} \cdot \frac{(-1)^{i}}{2^{i}} \\
& =\frac{2 a+b}{3}-\left(\frac{a-b}{6}\right) \cdot \frac{(-1)^{i}}{2^{i}} \\
& =\frac{1}{3}\left[(2 a+b)+\frac{(-1)^{i+1}}{2^{i+1}} \cdot(a-b)\right] \\
& =\frac{1}{3}\left[\left(2 a+\frac{(-1)^{i+1}}{2^{i+1}} a\right)+\left(b+\frac{(-1)^{i}}{2^{i+1}} b\right)\right]
\end{aligned}
$$

Thus, by solving the recurrence relation on $c_{i}$ obtained by $D M R W$, Eqn. (1) is proved. For Case-II, i.e., $C_{h}$ is mixed with $C_{0}$ to produce $C_{1}$, we obtain $c_{1}=\frac{1}{2}\left(\frac{a+b}{2}+b\right)=\frac{a+3 b}{4}$ as ini- 
tial conditions; by solving the recurrence relation of Eqn. (3) Eqn. (2) can then be proved similarly. This completes the proof.

For IDMA, it is not straightforward to obtain a relation between the initial $C F \mathrm{~s}\left(C_{\ell}\right.$ and $\left.C_{h}\right)$ and the number of waste droplets $(W)$. Note that the number of sequential mix-split steps $\left(m^{\prime}\right)$ required by $I D M A$ to achieve the target $C F C_{t}$ starting from two initial boundary $C F$ s $C_{\ell}=\frac{a}{2^{d}}$ and $C_{h}=\frac{b}{2^{d}}$ of a fluid, where ${ }_{485} 0 \leq C_{\ell} \leq C_{t}<C_{h} \leq 1$, is $\left(\left\lfloor\log _{2}(b-a)\right\rfloor-1\right)$. Again, the total number of waste droplets generated is $W=\sum_{i=1}^{m^{\prime}} W(i)$. Also, for any mix-split step, $i, W(i)$ is either 1 or 0 as a $(k: k)$ mix-split model is assumed.

If $C_{\ell}=\frac{0}{2^{d}}$ (buffer solution with $0 \%$ conc.) and $C_{h}=\frac{2^{d}}{2^{d}}$ (sample fluid with $100 \%$ conc.), then Eqn. (1) and Eqn. (2) are $_{520}$ reduced to:

$$
c_{i}=\frac{2^{d}}{3}\left[\left(1+\frac{(-1)^{i}}{2^{i+1}}\right)\right]
$$

and

$$
c_{i}=\frac{2^{d}}{3}\left[\left(2+\frac{(-1)^{i+1}}{2^{i+1}}\right)\right]
$$

respectively, where where $i$ is a non-negative integer between 0 and $\left(\left\lfloor\log _{2} 2^{d}\right\rfloor-1\right)$, i.e., $(d-1)$.

Fig. 3(d) depicts the digraph for target $C F \frac{341}{1024}$ indicating $10(1: 1)$ mix-split steps and the intermediate $C F$ s as $C_{0}=\frac{512}{1024}$, $C_{1}=\frac{256}{1024}, C_{2}=\frac{384}{1024}, C_{3}=\frac{320}{1024}, C_{4}=\frac{352}{1024}, C_{5}=\frac{336}{1024}, C_{6}=$ $\frac{344}{1024}, C_{7}=\frac{340}{1024}, C_{8}=\frac{342}{1024}$ and $C_{9}=\frac{341}{1024}$. These $C F$ values can be obtained from Eqn. (5) by setting $d=10$ and $i=0,1, \ldots, 9$. In order to generate all these target $C F \mathrm{~s}$, only one droplet will be wasted. Similar target $C F \mathrm{~s}$ for which $W=1$, can be found from Eqn. (6) by putting $d=10$ and $i=0,1, \ldots, 9$. The result stated in Theorem 1 can now be used to reduce the production of waste droplets $(W)$ in the dilution process by modifying the mix-split sequence of $D M R W$. When the skew of a node in the mix-split graph of $D M R W$ is greater than two, we attempt to generate two intermediate $C F \mathrm{~s} C_{\ell}^{\prime}$ and $C_{h}^{\prime}$, from the two input $C F$ s $C_{\ell}$ and $C_{h}$, so that the $\operatorname{target} C F C_{t}$ is (nearly) equal to onethird (i.e., $\frac{1}{3}$ for Case-I) or two-third (i.e., $\frac{2}{3}$ for Case-II) of the interval $\left(C_{h}^{\prime}-C_{\ell}^{\prime}\right)$. We refer to this rule as the One-Third Rule, and use it in the proposed scheme IDMA (Algorithm 3 ) for the reduction of wastage in the dilution process.

\subsection{Algorithm Overview: IDMA}

In the proposed scheme IDMA, first ImproveDigraph is invoked, and then the One-Third Rule is applied iteratively to further reduce the total number of waste droplets $(W)$ generated in $D M R W$ [12], by controlling the skew of an intermediate node. However, we have observed that it may not be easy to determine a suitable interval of two $C F$ s such that the target $C F$ is (nearly) equal to one-third or two-third of the interval. Hence, we mimic the one-third rule in a slightly different way while implementing IDMA.

For any target $C F C_{t}$ with a desired accuracy level of $d$ and the supply of two input $C F \mathrm{~s} C_{\ell}$ and $C_{h}$, IDMA first constructs the modified digraph $M^{\prime}$ by calling ImproveDigraph (Algorithm 1). If in $M^{\prime}$, if there is any node whose skew value is more than two, or appears later compared to that in the mix-split sequence of the previous graph $M$, two new boundary $C F \mathrm{~s}$ are again determined based on the one-third rule. IDMA iterates

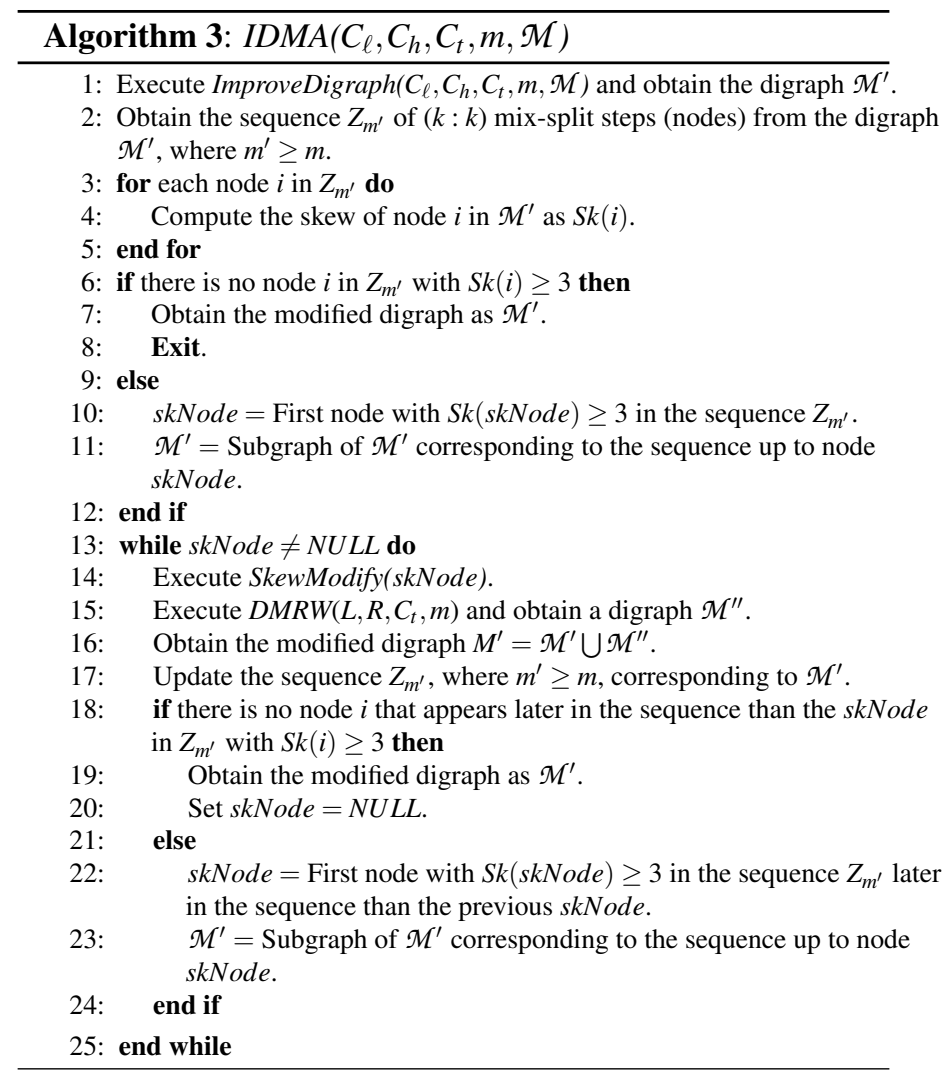




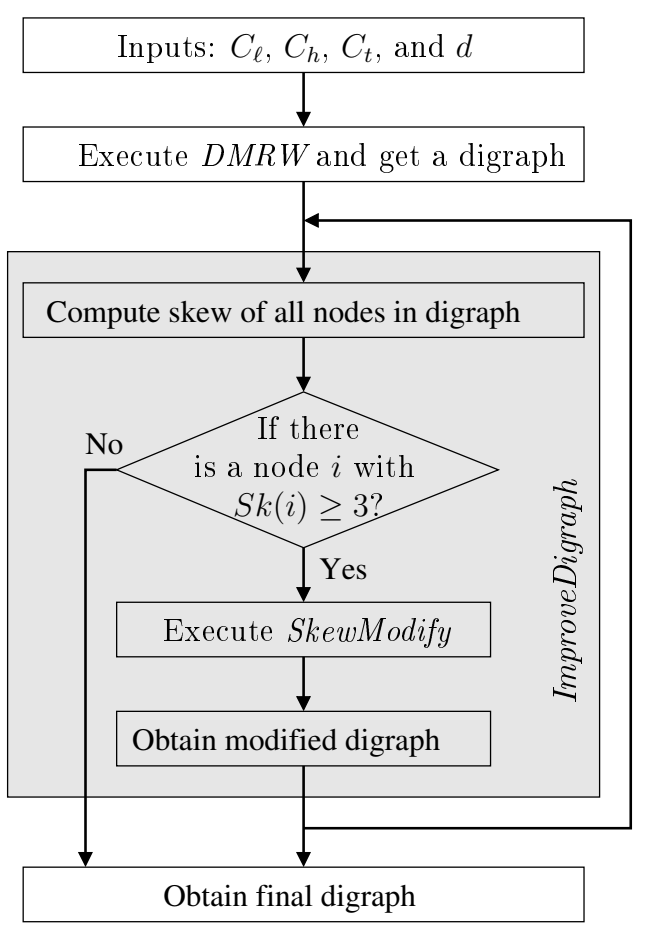

Figure 6: Flowchart of the proposed method IDMA.

this process to modify the digraph $\mathcal{M}^{\prime}$ obtained in the previous iteration, and whenever it finds a node in the digraph with $S k() \geq 3$, the procedure SkewModify() is called to modify the digraph. A flowchart of IDMA is shown in Fig. 6. The pseudocode of the proposed scheme IDMA is written as Algorithm 3

\subsection{Results of IDMA}

For a target $C F$, such as $\frac{1}{1024}$ or $\frac{1023}{1024}$, some nodes in the mixsplit digraph as obtained by ImproveDigraph, still have a high skew value. When IDMA is applied on the digraphs obtained by $D M R W$ for two target $C F \mathrm{~s} C_{t}=\frac{327}{1024}$ and $C_{t}=\frac{702}{1024}$, we obtain two modified digraphs as shown in Fig. 7(a) and Fig.7b), respectively. Note that for both these targets, IDMA further reduces $W$ as compared to DMRW or ImproveDigraph. For the target $C F \frac{327}{1024}$, the number of $(1: 1)$ mix-split steps $\left(T_{m s}\right)$ is also reduced from that of $D M R W$, whereas for the target $C F$ $\frac{702}{1024}, T_{m s}$ is reduced compared to ImproveDigraph.

We use several parameters to compare performances of four different dilution schemes, namely twoWayMix [11],

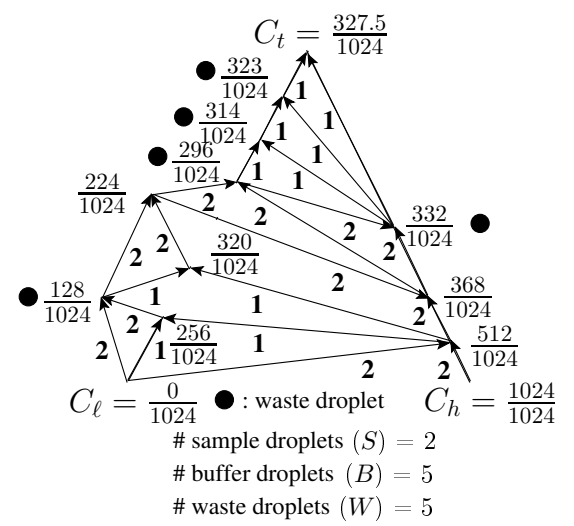

(a)

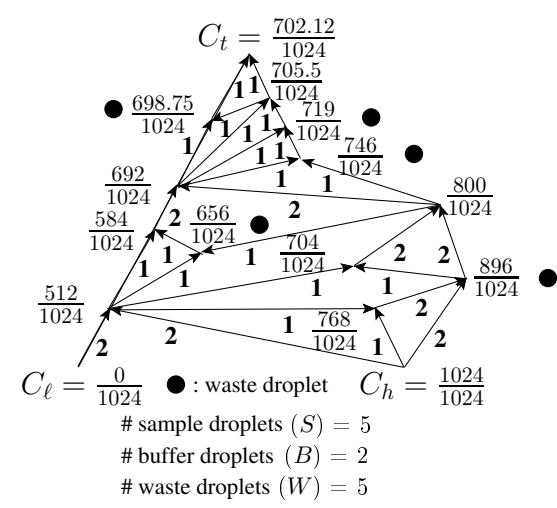

(b)

Figure 7: Digraphs obtained by IDMA for (a) $C_{t}=\frac{327}{1024}$ and (b) $C_{t}=\frac{702}{1024}$. Table 3: Comparative results of four methods for two target $C F \mathrm{~s} \frac{327}{1024}$ and $\frac{702}{1024}^{\dagger}$.

\begin{tabular}{|c|l|c|c|c|c|c|}
\hline Target $C F, C_{t}$ & Method & $W$ & $T_{m s}$ & $S$ & $B$ & $S k_{\max }$ \\
\hline \multirow{3}{*}{$\frac{327}{1024}$} & twoWayMix [1] & 9 & 10 & 5 & 6 & 1 \\
& DMRW [12] & 7 & 18 & 3 & 6 & 4 \\
& REMIA [13] & 7 & 17 & 3 & 6 & 3 \\
& IDMA & 5 & 17 & 2 & 5 & 3 \\
\hline \multirow{3}{*}{1024} & twoWayMix [11] & 8 & 9 & 7 & 3 & 1 \\
& DMRW [12] & 7 & 16 & 6 & 3 & 5 \\
& REMIA [13] & 7 & 12 & 3 & 6 & 5 \\
& IDMA & 5 & 17 & 5 & 2 & 4 \\
\hline
\end{tabular}

${ }^{\dagger}$ In order to produce two target droplets, $W$ : total number of waste droplets generated, $T_{m s}$ : total number of $(1: 1)$ mix-split steps, $S$ : total number of droplets of sample fluid, $B$ : total number of droplets of buffer solution, $S k_{\max }$ : the maximum skew value of any node in the digraph.

ple (buffer) droplets required to produce one/two target droplets is denoted as $S(B)$. The maximum skew value of a node in a mix-split digraph is represented as $S k_{\max }$. Table 3 reports these parameters for two examples. 


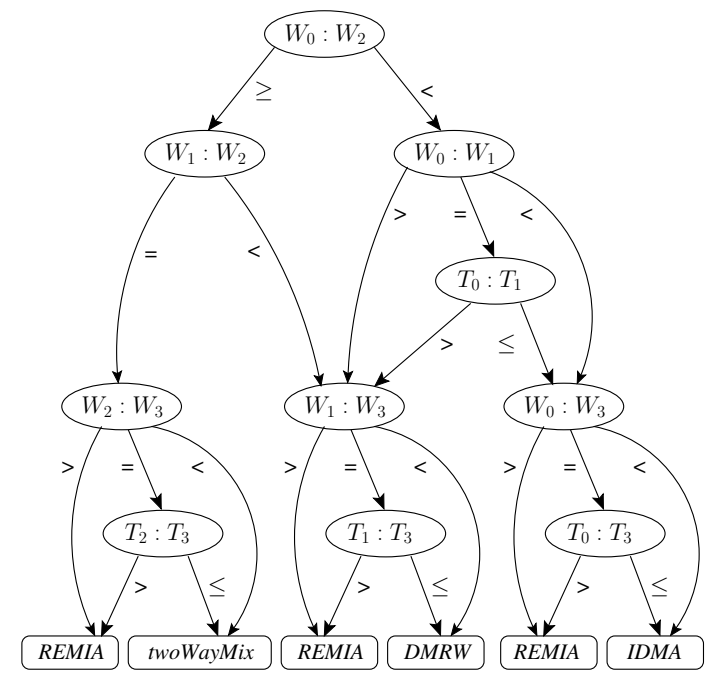

$W_{1} \leq W_{2}$ is always TRUE

$T_{2} \leq T_{3}, T_{1}, T_{0}$ is always TRUE

Figure 8: The decision tree followed in the integrated dilution scheme IDS.

\section{Integrated Scheme for Single-Target Dilution of a Fluid}

In this section, given target $C F C_{t}$, we present an Integrated ${ }_{575}$ Dilution Scheme $(I D S)$ that selects the best dilution algorithm among twoWayMix [11], DMRW [12], REMIA [13], and IDMA from the perspective of waste minimization. Let the total number of waste droplets $(W)$ for the digraph obtained by IDMA, DMRW [12], twoWayMix [11] and REMIA [13] be $W_{0}, W_{1}$, $W_{2}$ and $W_{3}$, respectively. Furthermore, the total number of (1:1) mix-split steps $\left(T_{m s}\right)$ in the sequence obtained by IDMA, $D M R W$ [12], twoWayMix [11] and REMIA [13] be $T_{0}, T_{1}, T_{2}$ and $T_{3}$, respectively. The following two theorems are immediate from the results stated in [11, 12].

Theorem 2. For every target $C F$, the number of waste droplets generated by DMRW is less than or equal to that produced by twoWayMix.

Theorem 3. For every target $C F$, the number of mix-split steps needed by DMRW is greater than or equal to that needed by ${ }^{590}$ twoWayMix.

From Theorem 2 and Theorem 3 , we observe that $W_{1} \leq W_{2}$ and $T_{1} \geq T_{2}$. Using a similar argument we can show that $T_{0} \geq$ $T_{2}$. The integrated scheme IDS aims to minimize waste $(W)$ as the primary objective and the total number of $(1: 1)$ mix-split 595 steps $\left(T_{m s}\right)$ as the second objective. A decision tree shown in

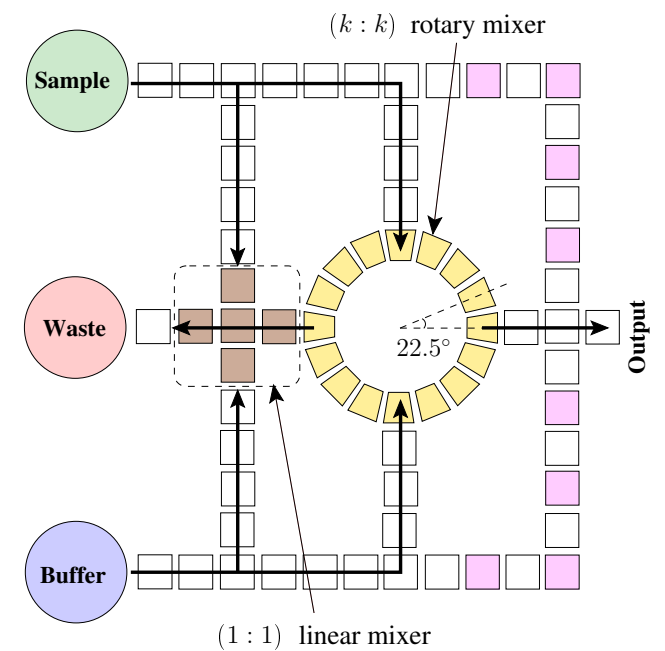

Figure 10: Layout of electrodes for the integrated scheme IDS.

Fig. 8, depicts the scheme. The complete procedure is shown as a flowchart in Fig. 9 .

\section{Proposed Layout for On-chip Dilution}

In order to expedite the dilution process by using $D M R W$ or IDMA, an on-chip ( $k: k)$-mixer is needed, $k \leq 5$, over the range of 1023 discrete target $C F$ s between $\frac{1}{1024}$ and $\frac{1023}{1024}$ [12]. It is observed that, when IDMA is chosen for 476 target $C F \mathrm{~s}, k$ is at most 3 and $D M R W$ is chosen for 526 target $C F$ s, where $k$ is at most 4; and for the remaining 21 cases where twoWayMix is chosen, where $k$ is at most 1 . This can be implemented using a rotary mixer [12, 31]. Our simulation experiments with IDS for $d=10$ reveal that among 1023 target $C F \mathrm{~s}, I D M A$ is chosen for 476 target $C F \mathrm{~s}$, where $k$ is at most 3, and $D M R W$ is chosen for 526 target $C F \mathrm{~s}$, where $k$ is at most 4; for the remaining 21 cases twoWayMix is chosen, where $k=1$. Hence, for singletarget dilution of a fluid, an architectural layout of electrodes with a rotary mixer as shown in Fig. 10 can be used to implement IDMA or DMRW. A portion of the same layout can be used as a three-electrode array (linear) mixer [32] to perform a homogeneous $(1: 1)$ mixing as well. droplet [32].

\subsection{Overheads of Linear and Rotary Mixers}

For providing a relative comparison of the algorithms, we normalize the workload of a rotary mixer with respect to a linear mixer. For a given target $C F$, the workload on the rotary 


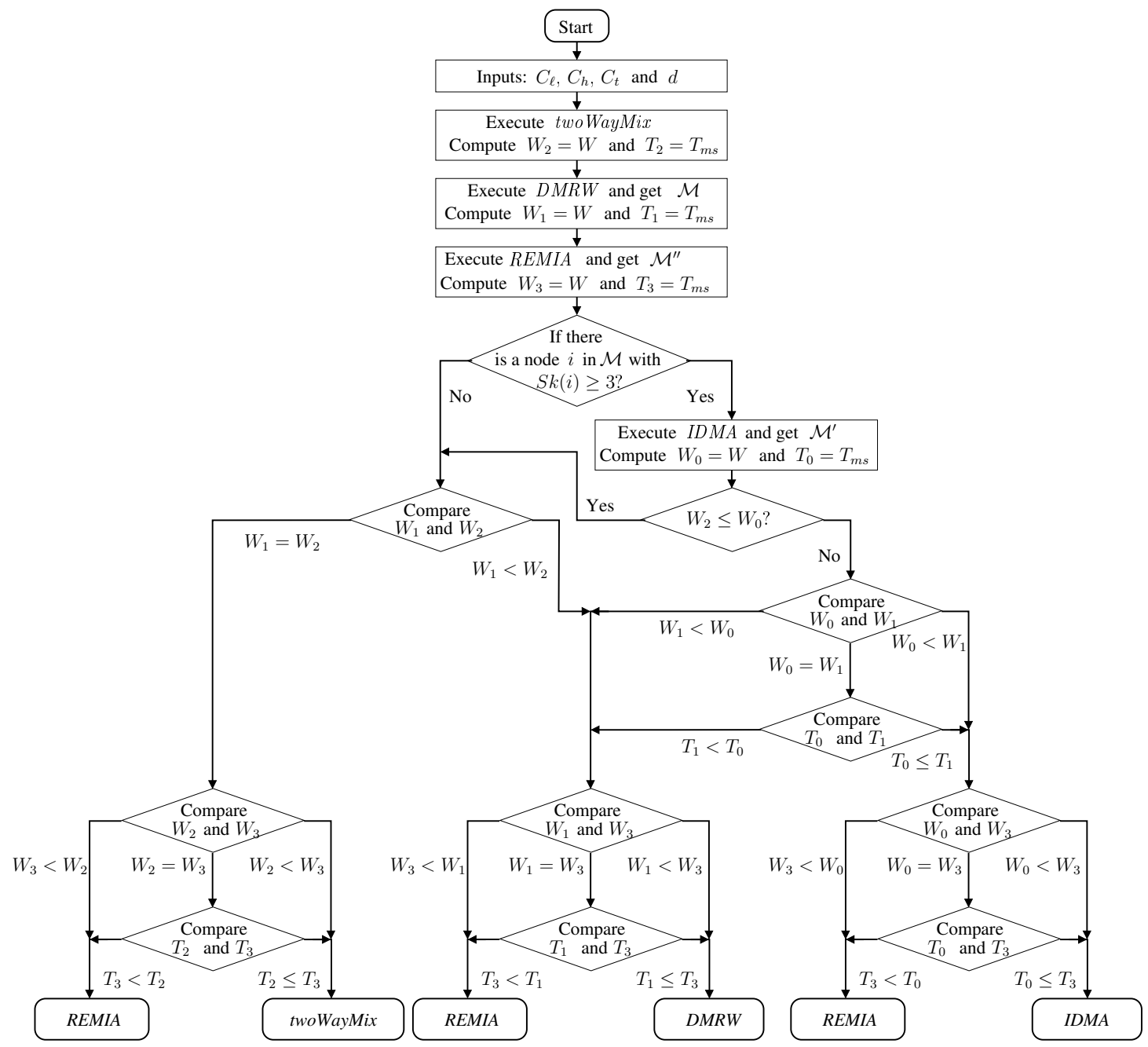

Figure 9: Flowchart of the integrated dilution scheme IDS.

mixer $\left(W L_{r}\right)$ is counted as $W L_{r}=\sum_{i} k$, where $i$ varies over all ( $k: k)$ mix-split steps with $k>1$ used in the sequence, i.e., how many times the rotary mixing is needed for $k>1$. The workload $\left(W L_{\ell}\right)$ on a linear mixer is counted as the number of ${ }_{615}$ (1:1) mix-split-step is needed in the sequence. For several target $C F \mathrm{~s}$, we report the mixer-workloads needed by different dilution schemes.

\subsection{Storage Unit Requirements}

Once an on-chip $(k: k)$ mix-split step $(k \geq 1)$ is executed by a linear or a rotary mixer, one or more droplets of the intermediate $C F$ so produced may need to be temporarily stored on some electrodes for subsequent use. However, the storage requirement depends on the target $C F$ as well the on the choice of dilution algorithm. Note that after a $(k: k)$ mix-split step, the volume of the mixture becomes $2 k$ units. In the next mix- split step, if only one $1 X$ droplet is used, we will need at most $(2 k-1)$ storage cells to store the rest of it.

In $D M R W$ or $I D M A$, only the two current boundary $C F \mathrm{~s}$ are needed at any instant of time, and their most recent values are obtained in the last two steps of the concerned mix-split sequence. Hence, in our simulation experiment, we observe the maximum storage requirement $(q)$ in two consecutive mixsplit steps over all target $C F$ values. We have observed that accuracy level $d=10$ is 6 . Based on this observation, we have designed our layout with on-chip storage electrodes (shown in 'pink' color) as in Fig. 10 .

For two target $C F \mathrm{~s} \frac{327}{1024}$ and $\frac{702}{1024}$, the values of $W L_{r}, W L_{\ell}$ and $q$ obtained by twoWayMix [11], DMRW [12], REMIA [13] and IDMA are shown in Table 4. 
Table 4: Comparative results of four methods for two target $C F \mathrm{~s} \frac{327}{1024}$ and $\frac{702}{1024}^{\dagger}$.

\begin{tabular}{|c|l|c|c|c|c|c|c|c|c|}
\hline $\begin{array}{c}\text { Target } \\
C F, C_{t}\end{array}$ & Method & $W$ & $T_{m s}$ & $S$ & $B$ & $S k_{\max }$ & $W L_{r}$ & $W L_{\ell}$ & $q$ \\
\hline \multirow{3}{*}{327} & twoWayMix [1] & 9 & 10 & 5 & 6 & 1 & 0 & 10 & $0^{65}$ \\
\hline 1024 & DMRW [12] & 7 & 18 & 3 & 6 & 4 & 13 & 5 & 5 \\
& REMIA [13] & 7 & 17 & 3 & 6 & 3 & 2 & 9 & 4 \\
& IDMA & 5 & 17 & 2 & 5 & 3 & 12 & 5 & 4 \\
\hline \multirow{3}{*}{702} & twoWayMix [11] & 8 & 9 & 7 & 3 & 1 & 0 & 9 & 0 \\
& DMRW [12] & 7 & 16 & 6 & 3 & 5 & 11 & 5 & 5 \\
& REMIA [13 & 7 & 12 & 3 & 6 & 5 & 5 & 7 & 5 \\
& IDMA & 5 & 17 & 5 & 2 & 4 & 8 & 9 & 3 \\
\hline
\end{tabular}

† In order to produce two target droplets, $W$ : total number of waste droplets generated, $T_{m s}$ : total number of $(1: 1)$ mix-split steps, $S$ : total number of droplets of sample fluid, $B$ : total number of droplets of buffer solution, $S k_{\max }$ : the maximum skew value of any node in the digraph, $W L_{r}$ : the workload on the rotary mixer, $W L_{\ell}$ : the workload on the linefig mixer, $q$ : total number of on-chip storage units required.

Theorem 4. At any instant of time during the execution of

DMRW or IDMA, intermediate-droplets of at most two distinct

CF values need to be stored. recent boundary values are needed, at any instant of time, during the execution of $D M R W$ or IDMA.

Based on this result, we allocate the storage electrodes in an arrangement of two non-overlapping pipelines (on the two opposite sides of the output of the rotary mixer), as shown in ${ }^{670}$ Fig. 10

\section{Simulation Results}

The simulation results of the proposed dilution algorithm $I D M A$, and the integrated dilution scheme IDS are reported in this section for an accuracy level of the desired $C F$ as $d=10$. It is observed that the maximum skew value $S k_{\max }$ in a digraph can be reduced by IDMA over $D M R W$. For a comparative study, we have computed the following performance parameters - the number of waste droplets $(W)$, the number of $(1: 1)$ mix-split steps $\left(T_{m s}\right)$, the maximum skew value of a node $\left(S k_{\max }\right)$ in the digraph, workload of the linear (rotary) mixer $W L_{\ell}\left(W L_{r}\right)$, and maximum on-chip storage requirement $(q)$.

\subsection{Test Data Set of Target CFs}

As mentioned earlier, we have conducted our simulation experiment with $I D S$ using all four dilution algorithms, namely twoWayMix [11], DMRW [12], REMIA [13] and IDMA, assuming $d=10$, i.e., all $1023 C F$ values are studied between $\frac{1}{1024}$ and $\frac{1023}{1024}$. A buffer (with $C F=0$ ) and the pure sample (with $C F=1)$ are assumed to be supplied as the two initial boundary fluids. The simulation results have been summarized in the following subsections.

\subsection{Performance of IDS}

Our results indicate that out of 1023 target $C F \mathrm{~s}, I D M A$ is most suitable for waste minimization and for reducing $T_{m s}$ for $435 C F$ s. Among the remaining $C F$ s $D M R W$ is found to be the best for 507 cases, whereas, REMIA performs best for other $61 C F \mathrm{~s}$ in terms of waste minimization. In the case of the remaining 20 target $C F$ s, twoWayMix can be used for waste minimization, as it requires the minimum number of $(1: 1)$ mixsplit steps, and is simple to implement. The pie-chart shown in Fig. 11 reflects this distribution of $C F \mathrm{~s}$ into four methods, twoWayMix, DMRW, REMIA and IDMA. In order to illustrate the choice made by $I D S$, we consider a range of target $C F$ s from $\frac{251}{1024}$ to $\frac{275}{1024}$ in Fig. 12 .

For some target $C F \mathrm{~s}$, the comparative results of four dilution methods are shown in Table 5 In the case of target $C F \mathrm{~s}$ $\frac{127}{1024}, \frac{251}{1024}, \frac{259}{1024}, \frac{263}{1024}, \frac{269}{1024}, \frac{313}{1024}, \frac{327}{1024}, \frac{513}{1024}$ and $\frac{702}{1024}, I D M A$ provides the best reduction in wastage and $T_{m s}$ compared to that by twoWayMix, DMRW or REMIA. Similarly, DMRW is the best suitable method for the target $C F \mathrm{~s}, \frac{261}{1024}, \frac{267}{1024}$ and $\frac{341}{1024}$ in waste minimization. For the target $C F \mathrm{~s} \frac{16}{1024}$ and $\frac{256}{1024}$, the best possible reduction in wastage with minimum $T_{m s}$ is obtained by twoWayMix. Whereas, REMIA is the best choice for the target $C F \mathrm{~s} \frac{264}{1024}$ and $\frac{268}{1024}$.

As IDMA performs best for $435 C F$ s out of 1023 target $C F$ s compared to twoWayMix, DMRW and REMIA, we report the comparative results of two important performance parameters $\left(W\right.$ and $\left.T_{m s}\right)$ in Fig. 13 (a) and 13 b), respectively, only for

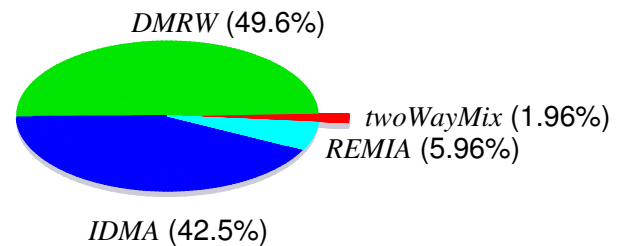

Figure 11: Distribution of 1023 target $C F$ s for waste minimization by four methods twoWayMix [11] (20 CFs), DMRW [12] (507 CFs), REMIA [13] (61 $C F \mathrm{~s})$ and IDMA (435 CF s). 


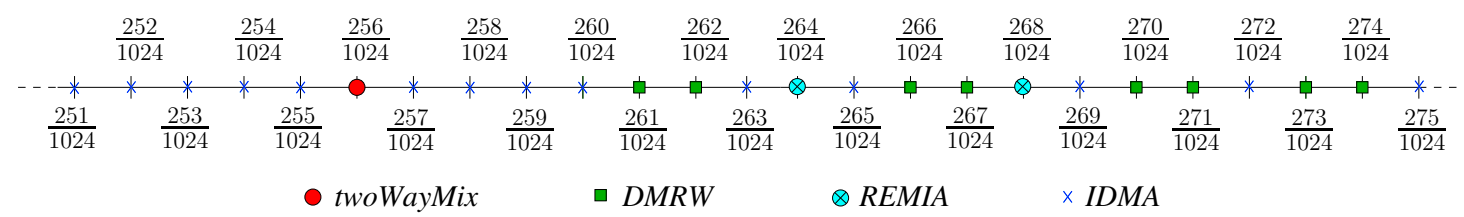

Figure 12: Selection of the best method among four methods twoWayMix [11], DMRW [12], REMIA [13] and IDMA, for different target $C F \mathrm{~s}$ in the range of ( $\frac{251}{1024}$ to $\frac{275}{1024}$ ) for waste minimization.

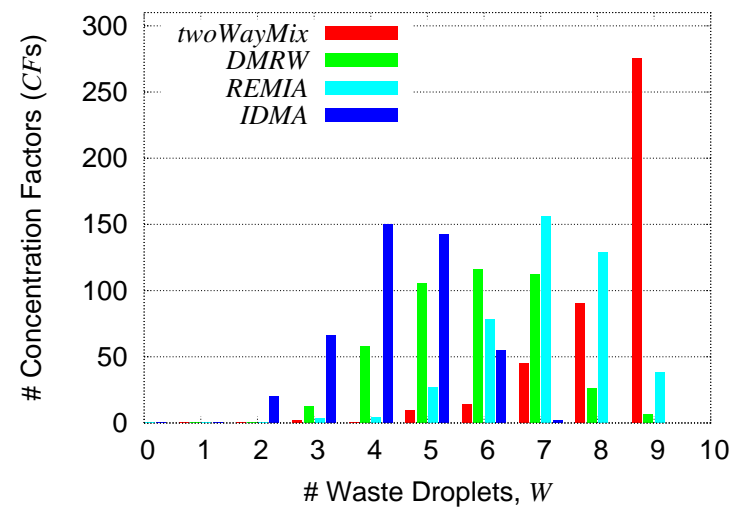

(a)

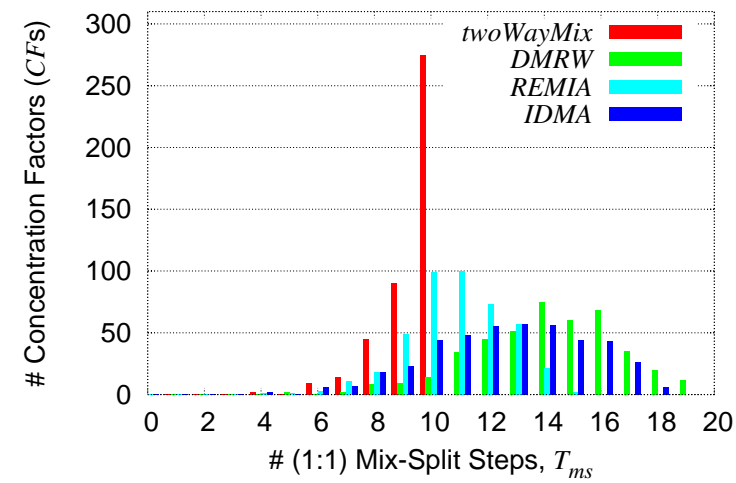

(b)

Figure 13: Histograms of four methods twoWayMix [11], DMRW [12], REMIA [13] and IDMA for $435 C F \mathrm{~s}$ out of 1023 target $C F$ s. Distribution of (a) $W$ values and (b) $T_{m s}$ values, with the count of target $C F \mathrm{~s}$.

Table 5: Selection of the best method among twoWayMix, DMRW, REMIA and $I D M A$ for waste minimization in case of some target $C F \mathrm{~s}$.

\begin{tabular}{|c|c|c|c|c|c|c|c|c|c|}
\hline \multirow{2}{*}{$\begin{array}{l}\text { Target } \\
C F, C_{t}\end{array}$} & \multicolumn{2}{|c|}{$I D M A$} & \multicolumn{2}{|c|}{$D M R W$ [12] } & \multicolumn{2}{|c|}{ twoWayMix 11} & \multicolumn{2}{|c|}{ REMIA [3] } & \multirow[t]{2}{*}{ Best Scheme } \\
\hline & $W_{0}$ & $T_{0}$ & $W_{1}$ & $T_{1}$ & $W_{2}$ & $T_{2}$ & $W_{3}$ & $T_{3}$ & \\
\hline$\frac{16}{1024}$ & 5 & 6 & 5 & 6 & 5 & 6 & 5 & 6 & twoWayMix \\
\hline$\frac{127}{1024}$ & 3 & 11 & 7 & 14 & 9 & 10 & 7 & 14 & $I D M A$ \\
\hline$\frac{251}{1024}$ & 5 & 17 & 6 & 13 & 9 & 10 & 7 & 13 & $I D M A$ \\
\hline$\frac{256}{1024}$ & 1 & 2 & 1 & 2 & 1 & 2 & 1 & 2 & twoWayMix \\
\hline$\frac{259}{1024}$ & 5 & 17 & 8 & 15 & 9 & 10 & 7 & 10 & $I D M A$ \\
\hline$\frac{261}{1024}$ & 5 & 17 & 5 & 13 & 9 & 10 & 7 & 10 & $D M R W$ \\
\hline$\frac{263}{1024}$ & 5 & 15 & 8 & 16 & 9 & 10 & 7 & 11 & $I D M A$ \\
\hline$\frac{264}{1024}$ & 5 & 16 & 5 & 10 & 6 & 7 & 5 & 7 & REMIA \\
\hline$\frac{267}{1024}$ & 5 & 16 & 5 & 13 & 9 & 10 & 6 & 10 & $D M R W$ \\
\hline$\frac{268}{1024}$ & 5 & 16 & 5 & 11 & 7 & 8 & 5 & 8 & REMIA \\
\hline$\frac{269}{1024}$ & 5 & 13 & 5 & 14 & 9 & 10 & 7 & 11 & $I D M A$ \\
\hline$\frac{313}{1024}$ & 5 & 15 & 7 & 18 & 9 & 10 & 6 & 11 & $I D M A$ \\
\hline$\frac{327}{1024}$ & 5 & 17 & 7 & 18 & 9 & 10 & 6 & 11 & $I D M A$ \\
\hline$\frac{341}{1024}$ & 1 & 10 & 1 & 10 & 9 & 10 & 5 & 10 & $D M R W$ \\
\hline$\frac{513}{1024}$ & 2 & 10 & 9 & 14 & 9 & 10 & 9 & 10 & IDMA \\
\hline$\frac{702}{1024}$ & 5 & 17 & 7 & 16 & 8 & 9 & 7 & 12 & $I D M A$ \\
\hline
\end{tabular}

these $435 C F$ s. Fig. 13 (a) reveals that IDMA can significantly

reduce the total number of waste droplets $(W)$ in producing a single-target dilution of a fluid compared to that by twoWayMix,
DMRW and REMIA. Moreover, Fig. 13, b) indicates that for several target $C F \mathrm{~s}, I D M A$ can further reduce the total number of (1:1) mix-split steps $\left(T_{m s}\right)$ required to produce a single-target 690 dilution in comparison with that by $D M R W$, while IDMA may require little more $T_{m s}$ compared to REMIA. Similar to DMRW, IDMA can trade-off wastage and the number of (1:1) mix-split steps.

\subsection{Performance Improvement by IDMA over DMRW}

Fig. 14(a) depicts that for several target $C F \mathrm{~s}, I D M A$ can further reduce $S k_{\max }$ values in the modified digraphs compared to that obtained by $D M R W$ [12]. For $435 C F \mathrm{~s}$, the maximum of $S k_{\max }$ values in a digraph obtained by $D M R W$ can be 9 , whereas IDMA can reduce this value to 5. However, in the case of twoWayMix, $S k_{\max }$ of a digraph is always one. Fig. 14(b) and 14(c) show that the mix-split sequence obtained by IDMA consists of more $(1: 1)$ operations compared to that needed in $D M R W$, and hence, the former reduces the workload on the rotary mixer. Hence, for many target $C F \mathrm{~s}, I D M A$ requires fewer number of storage units $(q)$ in comparison to $D M R W$, as evident from the simulation result shown in Fig. 14(d). 


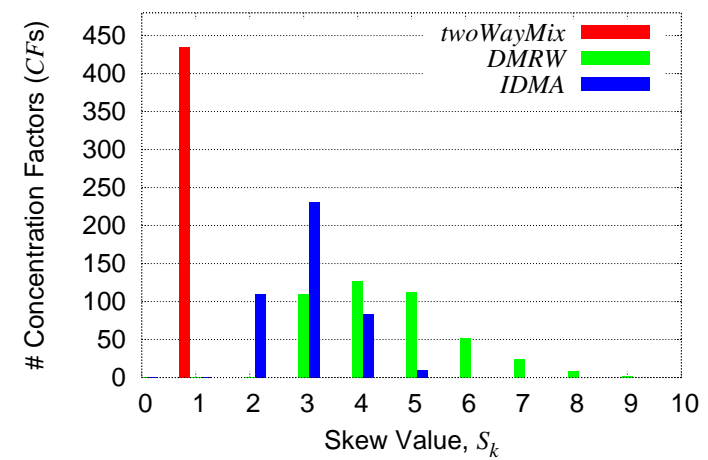

(a)

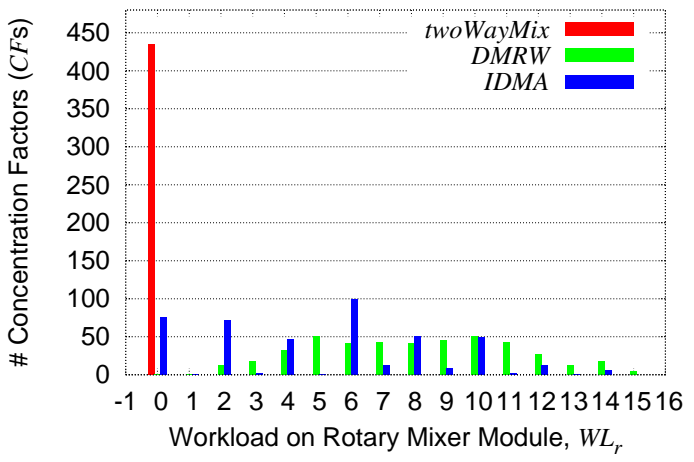

(c)

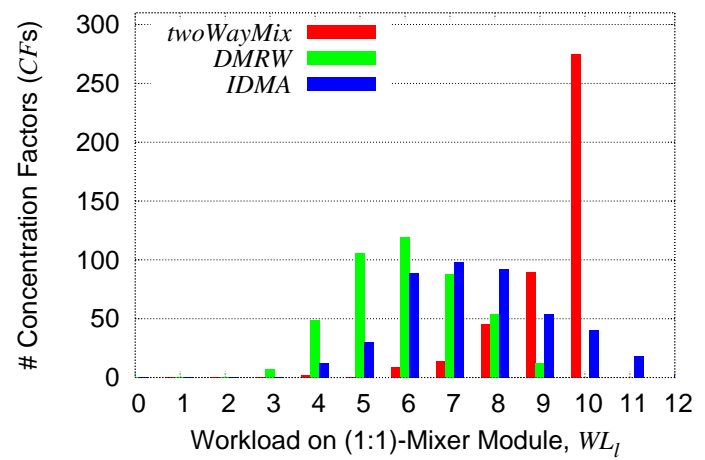

(b)

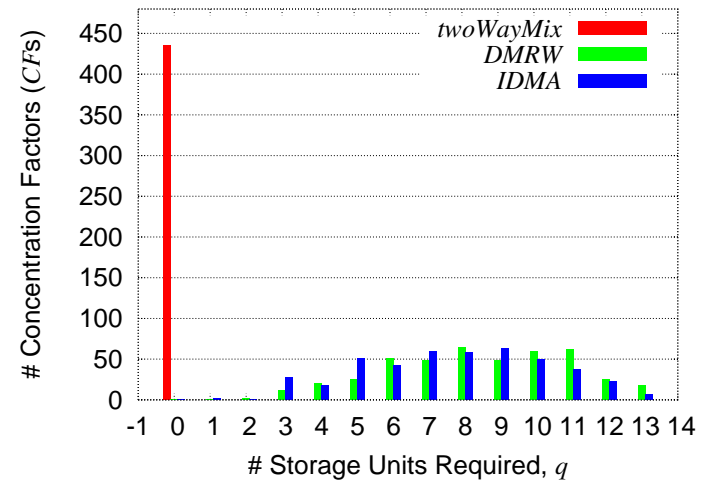

(d)

Figure 14: Histograms of three methods twoWayMix [11], DMRW [12] and IDMA for $435 C F$ s out of 1023 target $C F$ s. Distribution of (a) $S k_{\text {max }}$ values, (b) WL $L_{\ell}$ values, (c) $W L_{r}$ values, and (d) $q$ values, with the count of target $C F$ s.

\section{Conclusions}

In this paper, we have presented an improved algorithm called IDMA for reducing the total number of waste droplets while diluting a reactant fluid with a buffer fluid on a DMF biochip. We have also proposed an integrated dilution scheme IDS that can be used to select the best option among four stateof-the-art dilution algorithms for waste minimization. We have designed an architectural layout of electrodes that can support ample, in order to achieve a target $C F \frac{341}{1024}$ of a reactant fluid mix-split steps are required by twoWayMix, DMRW, or IDMA. However, just one $(2: 1)$ mix-split step is sufficient to produce the target droplets (if two droplets of the buffer fluid and one droplet of the reactant fluid are combined). We leave these issues for possible future work.

\section{Acknowledgements}

The authors wish to thank the Guest Editor, Dr. Evangeline Young, and the anonymous reviewers for their critical comments and valuable suggestions. This work of S. Roy was supported in part by Indian Institute of Technology Kharagpur and by Microsoft Research India PhD Fellowship (2010-2014). The work of P. P. Chakrabarti was supported in part by the Department of Science and Technology (DST), Government of India. The work of K. Chakrabarty was supported in part by the US National Science Foundation under grant CNS1135853. The work of B. B. Bhattacharya was supported by a special grant to Nanotechnology Research Triangle from Indian Statistical Institute, Kolkata. 


\section{References}

[1] S. Roy, B. B. Bhattacharya, K. Chakrabarty, Waste-Aware Dilution and ${ }_{785}$ Mixing of Biochemical Samples with Digital Microfluidic Biochips, in: Proc. of the Design, Automation Test in Europe Conference Exhibition (DATE), 2011, pp. 1059-1064.

[2] K. Chakrabarty, T. Xu, Digital Microfluidic Biochips: Design and Optimization, CRC Press, 2010.

[3] D. Jie, K. Chakrabarty, R. B. Fair, Scheduling of Microfluidic Operations for Reconfigurable Two-dimensional Electrowetting Arrays, IEEE Transactions on Computer-Aided Design of Integrated Circuits and System (TCAD) 20 (12) (2001) 1463-1468.

[4] H. Moon, A. R. Wheeler, R. L. Garrell, J. A. Loo, C.-J. Kim, An Integrated Digital Microfluidic Chip for Multiplexed Proteomic Sample Preparation and Analysis by MALDI-MS, Lab Chip 6 (9) (2006) 12131219.

[5] M. Abdelgawad, A. R. Wheeler, The Digital Revolution: A New Paradigm for Microfluidics, Advanced Materials 21 (8) (2009) 920-925.

[6] R. B. Fair, Digital Microfluidics: Is a True Lab-on-a-Chip Possible?, Microfluidics and Nanofluidics 3 (3) (2007) 245-281.

[7] M. G. Pollack, R. B. Fair, A. D. Shenderov, Electrowetting-Based Actuation of Liquid Droplets for Microfluidic Applications, Applied Physics Letters 77 (11) (2000) 1725-1726.

[8] H. Ren, V. Srinivasan, R. B. Fair, Design and Testing of an Interpolating Mixing Architecture for Electrowetting-Based Droplet-On-Chip Chemical Dilution, in: Proc. of the International Solid-State Sensors, Actuators and Microsystems Conference (TRANSDUCERS), 2003, pp. 619-622.

[9] M. J. Jebrail, A. R. Wheeler, Digital Microfluidic Method for Protein Extraction by Precipitation, Analytica Chimica Acta 81 (1) (2009) 330-335.

[10] W. C. Nelson, J. Y. Kim, Monolithic Fabrication of EWOD Chips for Picoliter Droplets, Journal of Microelectromechanical Systems 20 (6) (2011) 1419-1427.

[11] W. Thies, J. P. Urbanski, T. Thorsen, S. Amarasinghe, Abstraction Layers for Scalable Microfluidic Biocomputing, Natural Computing 7 (2) (2008) 255-275.

[12] S. Roy, B. B. Bhattacharya, K. Chakrabarty, Optimization of Dilution and Mixing of Biochemical Samples using Digital Microfluidic Biochips, IEEE Transactions on Computer-Aided Design of Integrated Circuits and Systems (TCAD) 29 (11) (2010) 1696-1708.

[13] J.-D. Huang, C.-H. Liu, T.-W. Chiang, Reactant Minimization during Sample Preparation on Digital Microfluidic Biochips using Skewed Mixing Trees, in: Proc. of the IEEE/ACM International Conference on Computer-Aided Design (ICCAD), 2012, pp. 377-384.

[14] E. J. Griffith, S. Akella, M. K. Goldberg, Performance Characterization of a Reconfigurable Planar-Array Digital Microfluidic System, IEEE Transactions on Computer-Aided Design of Integrated Circuits and Systems (TCAD) 25 (2) (2006) 345-357.

[15] D. Mitra, S. Roy, K. Chakrabarty, B. B. Bhattacharya, On-Chip Sample
Preparation with Multiple Dilutions using Digital Microfluidics, in: Proc. of the IEEE Computer Society Annual Symposium on VLSI (ISVLSI), 2012, pp. 314-319.

[16] S. Roy, B. B. Bhattacharya, S. Ghoshal, K. Chakrabarty, Low-Cost Dilution Engine for Sample Preparation using Digital Microfluidic Biochips, in: Proc. of the International Symposium on Electronic System Design (ISED), 2012, pp. 203-207.

[17] S. Roy, B. B. Bhattacharya, S. Ghoshal, K. Chakrabarty, HighThroughput Dilution Engine for Sample Preparation on Digital Microfluidic Biochips, IET Computers \& Digital Techniques (IET-CDT) 8 (2014) 163-171.

[18] S. Roy, B. B. Bhattacharya, S. Ghoshal, K. Chakrabarty, Optimal TwoMixer Scheduling in Dilution Engine on a Digital Microfluidic Biochip, in: Proc. of the International Symposium on Electronic System Design (ISED), 2013, pp. 82-86.

[19] S. Bhattacharjee, A. Banerjee, B. B. Bhattacharya, Sample Preparation with Multiple Dilutions on Digital Microfluidic Biochips, IET Computers \& Digital Techniques (IET-CDT) 8 (2014) 49-58.

[20] T. A. Dinh, S. Yamashita, T.-Y. Ho, A Network-Flow-Based Optimal Sample Preparation Algorithm for Digital Microfluidic Biochips, in: Proc. of the Asia and South Pacific Design Automation Conference (ASPDAC), 2014, pp. 225-230.

[21] Y.-L. Hsieh, T.-Y. Ho, K. Chakrabarty, On-Chip Biochemical Sample Preparation Using Digital Microfluidics, in: Proc. of the IEEE Biomedical Circuits and Systems Conference (BioCAS), 2011, pp. 297-300.

[22] Y.-L. Hsieh, T.-Y. Ho, K. Chakrabarty, A Reagent-Saving Mixing Algorithm for Preparing Multiple-Target Biochemical Samples Using Digital Microfluidics, IEEE Transactions on Computer-Aided Design of Integrated Circuits and Systems (TCAD) 31 (11) (2012) 1656-1669.

[23] T.-W. Chiang, C.-H. Liu, J.-D. Huang, Graph-Based Optimal Reactant Minimization for Sample Preparation on Digital Microfluidic Biochips, in: Proc. of International Symposium on VLSI Design, Automation, and Test (VLSI-DAT), 2013, pp. 1-4.

[24] C.-H. Liu, H.-H. Chang, T.-C. Liang, J.-D. Huang, Sample Preparation for Many-Reactant Bioassay on DMFBs using Common Dilution Operation Sharing, in: Proc. of the IEEE/ACM International Conference on Computer-Aided Design (ICCAD), 2013, pp. 615-621.

[25] S. Bhattacharjee, A. Banerjee, B. B. Bhattacharya, Multiple Dilution Sample Preparation Using Digital Microfluidic Biochips, in: Proc. of the International Symposium on Electronic System Design (ISED), 2012, pp. 188-192.

[26] J.-D. Huang, C.-H. Liu, H.-S. Lin, Reactant and Waste Minimization in Multitarget Sample Preparation on Digital Microfluidic Biochips, IEEE Transactions on Computer-Aided Design of Integrated Circuits and Systems (TCAD) 32 (10) (2013) 1484-1494.

[27] S. Roy, B. B. Bhattacharya, S. Ghoshal, K. Chakrabarty, An Optimal Two-Mixer Dilution Engine with Digital Microfluidics for Low-Power Applications, ASP Journal of Low Power Electronics (JOLPE) 10 (3) 
(2014) 1-12.

[28] D. Mitra, S. Roy, S. Bhattacharjee, K. Chakrabarty, B. B. Bhattacharya, On-Chip Sample Preparation for Multiple Targets Using Digital Microfluidics, IEEE Transactions on Computer-Aided Design of Integrated Circuits and Systems (TCAD) 33 (8) (2014) 1131-1144.

[29] S. Roy, B. B. Bhattacharya, S. Ghoshal, K. Chakrabarty, On-Chip Dilution from Multiple Concentrations of a Sample Fluid using Digital Microfluidics, in: Proc. of the International Symposium on VLSI Design and Test (VDAT), 2013, pp. 1-9.

840 [30] S. Roy, B. B. Bhattacharya, S. Ghoshal, K. Chakrabarty, Theory and Analysis of Generalized Mixing and Dilution of Biochemical Fluids Using Digital Microfluidic Biochips, ACM Journal on Emerging Technologies in Computing Systems (JETC) 11 (1) (2014) 2.1-2.33.

[31] Ring Structure: Digital Microfluidics by Electrowetting, Duke University, http://microfluidics.ee.duke.edu/videos/mpegs/rotary_flow.mpg (September 2009).

[32] P. Y. Paik, V. K. Pamula, R. B. Fair, Rapid Droplet Mixers for Digital Microfluidic Systems, Lab Chip 3 (4) (2003) 253-259. 